\title{
Modelagem estocástica para dinâmicas de colonização e colapso
}

\author{
Alejandro Roldan Correa
}

TESE APRESENTADA

$\mathrm{AO}$

Instituto de Matemática e EstatísticA

DA

Universidade de SÃo PaUlo

PARA

OBTENÇÃO DO TÍTULO

$\mathrm{DE}$

Doutor EM CiênCIAS

\author{
Programa: Estatística \\ Orientador: Prof. Dr. Fábio Prates Machado
}

Durante o desenvolvimento deste trabalho o autor recebeu auxílio financeiro da CAPES/CNPq

São Paulo, Fevereiro de 2016 


\section{Modelagem estocástica para dinâmicas de colonização e colapso}

Esta versão da tese contém as correções e alterações sugeridas pela

Comissão Julgadora durante a defesa da versão original do trabalho, realizada em 18/02/2016. Uma cópia da versão original está disponível no Instituto de Matemática e Estatística da Universidade de São Paulo.

Comissão Julgadora:

- Prof. Dr. Fábio Prates Machado-(Presidente) - USP.

- Prof. Dr. Luiz Renato Gonçalves Fontes - USP.

- Prof. Dr. Rinaldo Bruno Schinazi - UCCS.

- Prof. Dr. Élcio Lebensztayn - UNICAMP.

- Prof. Dr. Valdivino Vargas Junior - UFG. 
À memória de minha avó, Rosa Ofelia Roldan. 


\section{Agradecimentos}

Aos professores Fábio Machado e Rinaldo Schinazi pela confiança, apoio e orientação durante o desenvolvimento deste trabalho.

A todos os professores do IME-USP que tive o prazer de conhecer. Especialmente ao professor Luiz Renato Fontes pelos seus ensinamentos e o exemplo de profissionalismo.

A Cristian Coletti, Alexander Valencia e Valdivino Vargas pelos conselhos e motivação.

Aos meus colegas e amigos da pós-graduação pela companhia e interessantes discussões. Em particular, a Duvan Cataño, Elkin Cardenas, Nubia Esteban, Carolina Grejo e Maicon Pinheiro.

Aos meus pais e ao meu irmão pelo amor, incentivos e apoio incondicional.

A minha namorada Elizabeth pelo amor, dedicação e paciência ao longo destes anos.

À CAPES e CNPq (Processo 141046/2013-9) pelo apoio financeiro. 


\section{Resumo}

Roldan-Correa, A. Modelagem estocástica para dinâmicas de colonização e colapso. 2016. Tese (Doutorado) - Instituto de Matemática e Estatística, Universidade de São Paulo, São Paulo, 2016.

Algumas metapopulações de espécies, como formigas, vivem em colônias que crescem durante algum tempo e depois colapsam. Após o colapso poucos indivíduos sobrevivem. Esses indivíduos se dispersam tentando fazer novas colônias que podem ou não se estabelecer dependendo do ambiente que encontram. Recentemente, Schinazi [20] usou cadeias de nascimento e morte em ambientes aleatórios para modelar tais populações, e mostrou que a dispersão aleatória é uma boa estratégia para a sobrevivência da população. Nesta tese, introduzimos outros modelos estocásticos de colonização e colapso para os quais consideramos restrições espaciais e diferentes tipos de colapsos. Obtemos para esses novos modelos condições de sobrevivência e extinção. Debatemos algumas situações nas quais a dispersão nem sempre é uma boa estratégia de sobrevivência. Além disso, discutimos a relação destes modelos com outros conhecidos na literatura. Técnicas de percolação, acoplamento e comparação com processos de ramificação convenientemente definidos são usadas para obter os resultados aqui estabelecidos.

Palavras-chave: Metapopulação, percolação, acoplamento, processo de ramificação. 


\section{Abstract}

Roldan-Correa, A. Stochastic modeling for dynamics of colonization and collapse. 2016. Tese (Doutorado) - Instituto de Matemática e Estatística, Universidade de São Paulo, São Paulo, 2016.

Some metapopulations, such as ants, live in colonies that grow for a while and then collapse. Upon collapse, very few individuals survive. These individuals disperse, trying to establish new colonies that may or may not settle, depending on the environment they encounter. Recently, Schinazi [20] used birth and death chains in random environments to model such populations, and showed that random dispersion is a good strategy for the survival of the population. In this thesis, we introduce other stochastic models of colonization and collapse for which we consider spatial constraints and different kinds of collapse. We obtain conditions for survival and extinction in these new models. We discuss some situations in which dispersion is not always a good survival strategy. In addition, we discuss the relation of these models to others known in the literature. Percolation and coupling techniques and comparison with suitably defined branching processes are used to obtain the results set forth herein.

Keywords: Metapopulation, percolation, coupling, branching process. 


\section{Sumário}

1 Introdução 1

1.1 Considerações preliminares . . . . . . . . . . . . . . . . . 1

1.2 Estratégias de sobrevivência . . . . . . . . . . . . . . . . . 2

1.3 Contribuições e organização do trabalho . . . . . . . . . . . . . 3

2 Colonização e colapso $\quad \mathbf{5}$

2.1 Introdução . . . . . . . . . . . . . . . . . . . 5

2.2 Modelo espacial . . . . . . . . . . . . . . . . . . . 5

2.2.1 Construção gráfica de $C C(\mathcal{G}, \lambda, p) \ldots \ldots \ldots$

2.2 .2 Sobrevivência e extinção de $C C(\mathcal{G}, \lambda, p) \ldots \ldots \ldots$

2.3 Resultados principais . . . . . . . . . . . . . . . . 11

2.4 Modelos não espaciais . . . . . . . . . . . . . . . . . . . . . . . . . . 12

2.5 Provas . . . . . . . . . . . . . . . . . . . . 15

2.5.1 Resultados auxiliares . . . . . . . . . . . . 15

2.5.2 Provas dos resultados principais . . . . . . . . . . 17

3 Dispersão como estratégia $\quad 22$

3.1 Introdução . . . . . . . . . . . . . . . . . . . . . . 22

3.2 Modelos de crescimento . . . . . . . . . . . . . . . . . . . . 22

3.2.1 Modelo sem dispersão . . . . . . . . . . . . . . . . . . 23

3.2.2 Modelo com dispersão e sem restrições espaciais . . . . . . . . . 24

3.2.3 Modelo com dispersão e restrições espaciais . . . . . . . . . . . . 26

3.3 Dispersão como uma estratégia de sobrevivência . . . . . . . . . . . . . 27

3.4 Provas . . . . . . . . . . . . . . . . . . . . . . . . . . . . . . 29

$\begin{array}{ll}\text { Referências Bibliográficas } & 38\end{array}$ 


\section{Capítulo 1}

\section{Introdução}

\subsection{Considerações preliminares}

Modelos metapopulacionais referem-se em geral a populações espacialmente estruturadas em populações locais que estão conectadas via migrações. Cada população local evolui sem estrutura espacial, podendo aumentar ou diminuir, sobreviver, morrer ou migrar do seu local de maneiras diferentes. Diversos fenômenos biológicos influenciam a dinâmica de uma metapopulação. As espécies podem adotar diferentes estratégias para melhorar a sua probabilidade de sobrevivência, no entanto, outros fatores podem favorecer a sua extinção. Uma pergunta comum para a maioria das pesquisas feitas neste campo é por que algumas espécies sobrevivem, enquanto outras no mesmo ecossistema morrem. Ver Hanski [11] para saber mais sobre metapopulações.

As populações biológicas estão frequentemente expostas a eventos catastróficos que causam remoção em massa: Epidemias, intervenções humanas, desastres naturais, etc. A fim de explicar o comportamento dessas populações, diversos modelos estocásticos têm sido propostos (para referências ver Kapodistria et al. [14]). A maioria desses modelos são tratados no âmbito dos processos de Markov a tempo contínuo. Em particular, processos de nascimento e morte e suas generalizações têm sido amplamente estudados. Esses modelos se concentram no cálculo de importantes medidas como probabilidades de equilíbrio, probabilidades de extinção, tempo médio até a extinção, entre outras.

O mecanismo de catástrofe, em sua forma mais simples, remove instantaneamente toda a população (catástrofe total) cada vez que ocorre um evento catastrófico. Mais especificamente, o modelo de catástrofe total assume que os indivíduos são expostos a um efeito catastrófico que afeta massivamente toda a população e conduz à extinção. 
No entanto, na maioria das situações práticas isso não acontece, portanto, é natural supor que uma catástrofe elimina só uma parte da população. Com a hipótese de que os indivíduos de uma população são expostos simultaneamente a um efeito catastrófico, é adequado assumir catástrofes binomiais. O modelo de catástrofe binomial assume que nos tempos de catástrofe cada indivíduo sobrevive com probabilidade $p \in(0,1)$, independentemente de qualquer outra coisa. Modelos com catástrofes binomiais foram introduzidas por Brockwell et al. [6] e têm sido bastante estudados, veja por exemplo, Artalejo et al.[1], Kapodistria et al.[14] e as referências aí contidas.

$\mathrm{Na}$ literatura de sistemas de partículas interagentes, podemos encontrar exemplos interessantes de modelos biológicos com uma estrutura espacial e que estão sujeitos a catástrofes. Há um certo número de modelos, por exemplo, Belhadji e Lanchier [3], Borrello [5], Kang et al. [13], Krone [15], Schinazi [21, 22], que são ou podem ser interpretados como modelos demográficos. Cada sítio de $\mathbb{Z}^{d}$ é ocupado por um grupo de indivíduos que estão sujeitos a catástrofes. O efeito de cada catástrofe remove parte ou a totalidade dos indivíduos presentes em um sítio dado. Isto proporciona uma classe importante de modelos estocásticos, incluindo catástrofes que eliminam apenas uma parte da população.

\subsection{Estratégias de sobrevivência}

Muitas espécies, como as formigas, vivem em colônias que crescem durante algum tempo e depois colapsam. Após o colapso poucos indivíduos sobrevivem. Esses indivíduos se dispersam tentando fazer novas colônias que podem ou não se estabelecer dependendo do ambiente que encontram. Recentemente, Schinazi [20] propôs um modelo estocástico não espacial para modelar tal tipo de dinâmica populacional e mostrou que a dispersão aleatória é uma boa estratégia para a sobrevivência da população. O modelo e os principais resultados em Schinazi [20] estabelecem o seguinte.

Teorema 1.2.1 (Teorema $1 \mathrm{em}[20]$ ). Considere um processo no qual indivíduos estão divididos em um número aleatório de colônias independentes. Cada colônia é um processo de nascimento e morte, onde a taxa de nascimento é amostrada de uma distribuição fixada $\mu$ e a taxa de morte é 1. Cada colônia é associada a um tempo aleatório $T$ amostrado de uma distribuição fixada $\nu$. No tempo $T$ cada indivíduo na colônia começa uma nova colônia independente com um novo $\lambda$ e um novo T. Esta população sobrevive se e somente se $\mathbb{E}[\exp ((\Lambda-1) \tau)]>1$, onde $\Lambda$ tem distribuiçãa $\mu$ e $\tau$ tem distribuição $\nu$.

Observe que neste modelo um colapso representa a destruição do habitat onde a 
colônia se encontra (indivíduos não morrem no colapso) provocando a migração dos seus indivíduos para outros locais. A fim de modelar o fato que muitos indivíduos morrem quando uma colônia colapsa, o Teorema 1.2.1 é particularmente interessante para distribuições nas quais $\mu([0,1))$ é quase 1 , isto faz que muitos dos $\lambda$ 's amostrados sejam menores que 1 e as novas colônias colapsem rapidamente.

Para uma cadeia de nascimento e morte (clássica) com taxa de nascimento fixa $\mathbb{E}(\Lambda)$ e taxa de morte 1 é conhecido que a cadeia morre se $\mathbb{E}(\Lambda) \leq 1$, ver Allen [2]. Em contrapartida, no modelo de Schinazi, $\mathbb{E}(\Lambda)$ pode ser arbitrariamente próximo de 0 e ainda ter sobrevivência (ver exemplo 1.1 em [20]). Assim, comparando com o modelo de ambiente fixo (sem colapsos), segue que a dispersão aleatória ajuda a sobrevivência. Porém, não é claro se a sobrevivência se torna mais provável pela multiplicação de ambientes aleatórios (dispersão) ou simplesmente devida à mudança do ambiente. Para clarear tal ideia, Schinazi [20] introduz outro modelo sem dispersão e com mudanças globais do ambiente para toda a população. O modelo é descrito no seguinte Teorema.

Teorema 1.2.2 (Teorema 2 em [20] ). Considere uma cadeia de nascimento e morte em ambientes aleatórios para a qual a taxa de morte é 1 e a taxa de nascimento muda nos tempos $T_{k}=\tau_{1}+\tau_{2}+\cdots+\tau_{k}$ onde $\tau_{1}, \tau_{2}, \ldots$ são variáveis aleatórias independentes e identicamente distribuídas. As taxas de nascimento são amostradas de uma distribuição fixada $\mu$. Assuma que $\mathbb{E}\left(\tau_{1}\right)<\infty$ e $\mathbb{E}(\Lambda)<\infty$ onde $\Lambda$ tem distribuição $\mu$. Assuma que as taxas de nascimento e os tempos $\tau_{i}$ são independentes. Então, o processo sobrevive se e somente se $\mathbb{E}(\Lambda)>1$.

Esse resultado mostra que a cadeia de nascimento e morte em ambientes aleatórios sobrevive se e somente se a cadeia de nascimento e morte (clássica) com taxa de nascimento $\mathbb{E}(\Lambda)$ sobrevive. Vê-se então que apenas mudanças globais no ambiente não favorecem à sobrevivência. Dessa forma, Schinazi conclui que é de fato a mistura da dispersão com a mudança do ambiente o que ajuda à sobrevivência.

\subsection{Contribuições e organização do trabalho}

Nesta tese, introduzimos outros modelos estocásticos de colonização e colapso para os quais consideramos restrições espaciais e diferentes tipos de colapsos. Obtivemos para esses novos modelos condições de sobrevivência e extinção. Debatemos algumas situações nas quais a dispersão nem sempre é uma boa estratégia de sobrevivência. Além disso, discutimos a relação destes modelos com outros conhecidos na literatura. 
A tese está organizada da seguinte forma.

No Capítulo 2 introduzimos um modelo estocástico espacial de colonização e colapso em grafos. Na dinâmica de tal modelo, consideramos colapsos do tipo catástrofes binomiais e assumimos que os indivíduos adotam a estratégia de dispersão, mesmo quando restrições espaciais complicam essa estratégia. Além disso, consideramos uma versão não espacial deste modelo, a qual tem uma estreita relação com um modelo introduzido em Brockwell [7] e que proporciona interessantes conclusões da dispersão como estratégia de sobrevivência.

No Capítulo 3, com base no modelo de Artalejo et al. [1] e seguindo as ideias do Capítulo 2, analisamos modelos de crescimento de populações sujeitas a colapsos (catástrofes binomiais e catástrofes geométricas). Para os modelos aqui introduzidos, questionamos a vantagem da dispersão como estratégia de sobrevivência em função das restrições espaciais e dos tipos de colapsos que afetam a população. 


\section{Capítulo 2}

\section{Colonização e colapso}

\subsection{Introdução}

Muitas espécies (como formigas) vivem em colônias que crescem durante algum tempo e depois colapsam. Após o colapso poucos indivíduos sobrevivem. Estes indivíduos se dispersam tentando fazer novas colônias que podem ou não se estabelecerem dependendo do ambiente que encontram. Recentemente, Schinazi [20] usou cadeias de nascimento e morte em ambientes aleatórios para modelar tais populações e mostrou que a dispersão aleatória é uma boa estratégia para a sobrevivência da população. Neste capítulo propomos um outro modelo estocástico de colonização e colapso, para o qual consideramos colapsos do tipo catástrofe binomial e restrições espaciais que dificultam a dispersão. Obtemos condições para a sobrevivência e extinção da população. Além disso, relações com outros modelos conhecidos são discutidas. ${ }^{1}$

Este capítulo está dividido em cinco seções. Na Seção 2 definimos um processo estocástico espacial para colonização e colapso e apresentamos algumas de suas propriedades. Na Seção 3 são estabelecidos os principais resultados. Na Seção 4 introduzimos uma versão não espacial do nosso modelo e comparamos este com outros modelos conhecidos na literatura. Finalmente, na Seção 5 nós provamos os resultados apresentados na Seção 3.

\section{$2.2 \quad$ Modelo espacial}

Denotamos por $\mathcal{G}=(V, E)$ um grafo conexo não orientado de grau localmente limitado, onde $V:=V(\mathcal{G})$ é o conjunto de vértices de $\mathcal{G}$, e $E:=E(\mathcal{G})$ é o conjunto de elos de $\mathcal{G}$. Vértices são considerados vizinhos se eles pertencem a um elo comum.

\footnotetext{
${ }^{1}$ Os resultados deste Capítulo estão reunidos em Machado et al. [18]
} 
O grau de um vértice $x$ é o número de elos que têm $x$ como um ponto final. Um grafo é localmente limitado, se todos os seus vértices têm grau finito. Um grafo é $k$-regular se todos seus vértices têm grau $k$. A distância $d(x, y)$ entre os vértices $x$ e $y$ é a quantidade mínima de elos pelos quais se deve passar a fim de ir de $x$ para $y$. Com o abuso usual de notação, por $\mathbb{Z}^{d}$ denotamos o grafo com o conjunto de vértices $\mathbb{Z}^{d} \mathrm{e}$ conjunto de elos $\left\{\left\langle\left(x_{1}, \ldots, x_{d}\right),\left(y_{1}, \ldots, y_{d}\right)\right\rangle:\left|x_{1}-y_{1}\right|+\ldots\left|x_{d}-y_{d}\right|=1\right\}$. Também, $\mathbb{T}^{d}, d \geq 2$, denota a árvore homogênea com grau $d+1$.

Introduzimos o modelo estocástico de colonização e colapso em um grafo $\mathcal{G}$ como segue. Cada vértice de $\mathcal{G}$ pode estar ocupado por uma colônia ou estar vazio. Cada colônia começa com um único indivíduo. O número de indivíduos na colônia no tempo $t$ é determinado por um processo de nascimento puro (processo de Yule) de taxa $\lambda \geq 0$. Cada colônia é associada com um tempo exponencial de média 1. Quando o tempo exponencial ocorre, a colônia colapsa e o vértice da colônia se torna vazio. No tempo do colapso cada indivíduo da colônia sobrevive com probabilidade $p>0$ ou morre com probabilidade $q=1-p$. Cada sobrevivente pula aleatoriamente para um dos vértices vizinhos. Se o vértice já está ocupado o indivíduo morre, se o vértice está vazio o indivíduo inicia nele uma nova colônia. Quando mais de um indivíduo salta para um mesmo vértice vazio, somente um é responsável pela formação da colônia nova; os demais morrem. Denotaremos este modelo por $C C(\mathcal{G}, \lambda, p)$.

O modelo $C C(\mathcal{G}, \lambda, p)$ é um processo de Markov a tempo contínuo com espaço de estados $\mathbb{N}^{V}$ e cuja evolução (estado no tempo $t$ ) é denotada por $\eta_{t}$. Para cada vértice $x \in V, \eta_{t}(x)=i$ significa que no tempo $t$ existem $i$ indivíduos no vértice $x$.

\subsubsection{Construção gráfica de $C C(\mathcal{G}, \lambda, p)$}

Mostramos agora uma construção gráfica para o processo de colonização e colapso $C C(\mathcal{G}, \lambda, p)$ para $\mathcal{G}=\mathbb{Z}$. As ideias aqui apresentadas podem ser estendidas para $\mathcal{G}$ arbitrário.

Considere uma coleção de processos de Poisson independentes

$$
\left\{N_{i}^{x}, C^{x}: x \in \mathbb{Z}, i \geq 1\right\}
$$

onde para cada $x \in \mathbb{Z}$ e $i \geq 1$, as taxas de $N_{i}^{x}$ e $C^{x}$, são respectivamente, $i \lambda$ e 1 . 
Considere também a coleção de sequências independentes

$$
\left\{\left(X_{m}^{x, j}, Y_{m}^{x, j}\right)_{m \geq 1}: x \in \mathbb{Z}, j \geq 1\right\}
$$

onde $\left(X_{m}^{x, j}, Y_{m}^{x, j}\right)_{m \geq 1}$ é uma sequência tal que $X_{1}^{x, j}, X_{2}^{x, j}, \ldots, Y_{1}^{x, j}, Y_{2}^{x, j}, \ldots$ são variáveis aleatórias independentes e identicamente distribuídas $U[0,1]$. A sequência $\left(X_{m}^{x, j}, Y_{m}^{x, j}\right)_{m \geq 1}$ é associada ao $j$-ésimo tempo de ocorrência do processo $C^{x}$. A construção gráfica ocorre na região espaço-tempo $\mathbb{Z} \times(0, \infty)$.

- Em um tempo de ocorrência de $N_{i}^{x}$ : Se o vértice $x$ está no estado $i$, então mudamos o estado de $x$ ao estado $i+1$.

- No $j$-ésimo tempo de ocorrência de $C^{x}$ : Considere a sequência $\left(X_{m}^{x, j}, Y_{m}^{x, j}\right)_{m \geq 1}$. Se o vértice $x$ está no estado $i$, então mudamos o estado de $x$ ao estado 0 , e consideramos

$$
(K, L)_{i, p}^{x, j}:=\left(\sum_{m=1}^{i} \mathbf{1}\left\{X_{m}^{x, j}<p, Y_{m}^{x, j}<1 / 2\right\}, \sum_{m=1}^{i} \mathbf{1}\left\{X_{m}^{x, j}<p, Y_{m}^{x, j}>1 / 2\right\}\right)
$$

- Caso $K=0$ e $L \geq 1$. Se o vértice $x+1$ está no estado 0 , então mudamos o estado de $x+1$ para o estado 1 .

- Caso $K \geq 1$ e $L=0$. Se o vértice $x-1$ está no estado 0, então mudamos o estado de $x-1$ para o estado 1 .

- Caso $K \geq 1$ e $L \geq 1$. Se os vértices $x-1$ e $x+1$ estão no estado 0 , então mudamos os estados de $x-1$ e $x+1$ para o estado 1 . Se o vértice $x+1$ está em um estado diferente de 0 e o vértice $x-1$ está no estado 0 , então mudamos o estado de $x-1$ para o estado 1 . Se o vértice $x-1$ está em um estado diferente de 0 e o vértice $x+1$ está no estado 0 , então mudamos o estado de $x+1$ para o estado 1 .

Observe que $K$ e $L$ representam o número de indivíduos sobreviventes ao colapso que tencionam fundar uma nova colônia em $x-1$ e $x+1$, respectivamente.

Usando esta construção gráfica para o processo $C C(\mathbb{Z}, \lambda, p)$ é possível estabelecer a seguinte propriedade de monotonia do modelo.

Proposição 2.2.1. Seja $\eta_{t}$ um processo $C C(\mathbb{Z}, \lambda, p)$ e $\left|\eta_{t}\right|:=\sum_{x \in \mathbb{Z}} \eta_{t}(x)$. Então, a probabilidade

$$
\mathbb{P}\left(\left|\eta_{t}\right| \geq 1, \text { para todo } t \geq 0\right)
$$

é uma função não decrescente em $\lambda$ e não decrescente em p. 
Demonstração. É suficiente provar que é possível construir no mesmo espaço de probabilidade dois processos de colonização e colapso $\eta_{1, t}$ e $\eta_{2, t}$ de parâmetros $\left(\lambda_{1}, p_{1}\right)$ e $\left(\lambda_{2}, p_{2}\right)$, respectivamente, tais que $\lambda_{1} \leq \lambda_{2}, p_{1} \leq p_{2}$ e $\eta_{1,0} \leq \eta_{2,0}$ implica $\eta_{1, t} \leq \eta_{2, t}$ para todo $t>0$.

Como fizemos na construção gráfica, construímos o processo $\eta_{2, t}$ definindo uma coleção de processos de Poisson independentes

$$
\left\{N_{2, i}^{x}, C_{2}^{x}: x \in \mathbb{Z}, i \geq 1\right\}
$$

onde as taxas de $N_{2, i}^{x}$ e $C_{2}^{x}$, são respetivamente, $i \lambda_{2}$ e 1 . E consideramos uma coleção de sequências independentes

$$
\mathcal{C}_{2}=\left\{\left(X_{m}^{x, j}, Y_{m}^{x, j}\right)_{m \geq 1}: x \in \mathbb{Z}, j \geq 1\right\}
$$

onde $\left(X_{m}^{x, j}, Y_{m}^{x, j}\right)_{m \geq 1}$ é uma sequência tal que $X_{1}^{x, j}, X_{2}^{x, j}, \ldots, Y_{1}^{x, j}, Y_{2}^{x, j}, \ldots$ são variáveis aleatórias independentes e identicamente distribuídas $U[0,1]$. Isto gera um processo $\eta_{t}^{2}$ com as taxas adequadas.

Para construir $\eta_{1, t}$ podemos usar os mesmos processos Poisson $\left\{N_{2, i}^{x}\right\}$ para obter processos Poisson $\left\{N_{1, i}^{x}\right\}$ com taxas $i \lambda_{1}$ fazendo o seguinte. Cada vez que existe uma ocorrência para $N_{2, i}^{x}$, haverá uma ocorrência para $N_{1, i}^{x}$ com probabilidade $\lambda_{1} / \lambda_{2}$. Isto gera processos Poisson $\left\{N_{1, i}^{x}\right\}$ de taxas $i \lambda_{1}$. Além disso, definimos $C_{1}^{x}:=C_{2}^{x}$ e $\mathcal{C}_{1}:=\mathcal{C}_{2}$. Da construção gráfica, a coleção $\left\{N_{1, i}^{x}, C_{1}^{x}: x \in \mathbb{Z}, i \geq 1\right\}$ e a coleção $\mathcal{C}_{1}$ geram um processo $\eta_{1, t}$ com as taxas adequadas.

Pela construção dos processos $\eta_{1, t}$ e $\eta_{2, t}$ no mesmo espaço de probabilidade e do fato que $(K, L)_{i, p}^{x, j}$ é não decrescente em $i$ e não decrescente em $p$, vemos que se $\eta_{1,0} \leq \eta_{2,0}$ então nenhuma transição pode fazer o processo $\eta_{1, t}$ ter mais indivíduos que o processo $\eta_{2, t}$ em qualquer vértice dado, isto é, $\eta_{1, t} \leq \eta_{2, t}$.

Não é difícil observar que a Proposição 2.2.1 vale também para $\mathcal{G}$ arbitrário.

\subsubsection{Sobrevivência e extinção de $C C(\mathcal{G}, \lambda, p)$}

Entre os nossos principais objetivos está caracterizar a sobrevivência e a extinção do modelo $C C(\mathcal{G}, \lambda, p)$ segundo as seguintes definições.

Definição 2.2.1. Seja $\eta_{t}$ um processo $C C(\mathcal{G}, \lambda, p)$ começando com um número finito 
de colônias e $\left|\eta_{t}\right|:=\sum_{x \in V} \eta_{t}(x)$. Dizemos que $\eta_{t}$ sobrevive (globalmente) se

$$
\mathbb{P}\left(\left|\eta_{t}\right| \geq 1 \text { para todo } t \geq 0\right)>0 \text {. }
$$

Caso contrário, dizemos que o processo morre (globalmente).

Observação 2.2.1. Se o processo $\eta_{t}$ começa com um número infinito de colônias, então $\mathbb{P}\left(\left|\eta_{t}\right| \geq 1\right.$ para todo $\left.t \geq 0\right)=1$. De fato, suponha que no tempo 0 , os vértices $x_{1}, x_{2}, \ldots$ (infinitos) possuem colônia. Pela independência dos tempos de colapso segue que para cada $t>0$ e $n \geq 1$, os eventos $A_{n}(t):=\left\{\eta_{s}\left(x_{n}\right)>0, \forall 0 \leq s \leq t\right\}$ são independentes. Além disso,

$$
\sum_{n \geq 1} \mathbb{P}\left[A_{n}(t)\right]=\sum_{n \geq 1} e^{-t}=\infty
$$

donde pelo Lema de Borel-Cantelli segue que $\mathbb{P}\left[A_{n}(t)\right.$ para infinitos $\left.n\right]=1$. Portanto, quando o processo $\eta_{t}$ começa com um número infinito de colônias, sobrevive com probabilidade 1. Mesmo assim, pode existir morte local no sentido da seguinte definição.

Definição 2.2.2. Seja $\eta_{t}$ um processo $C C(\mathcal{G}, \lambda, p)$. Dizemos que $\eta_{t}$ morre localmente se para todo vértice $x \in V$ existe um tempo (aleatório) finito $T$ tal que $\eta_{t}(x)=0$ para todo $t>T$. Caso contrário, dizemos que o processo sobrevive localmente.

Dito de outra forma, morte local significa que todo vértice é colonizado somente um número finito de vezes.

Observação 2.2.2. Morte global implica morte local. Embora, a recíproca nem sempre é verdadeira. Considere, por exemplo, $\eta_{t}$ um processo $C C\left(\mathbb{Z}^{3}, 0,1\right) \operatorname{com}\left|\eta_{0}\right|=1$; neste caso $\eta_{t}$ pode ser visto como um passeio aleatório simétrico em $\mathbb{Z}^{3}$, o qual é transiente (ver Batachyara [4]). Portanto, $\eta_{t}$ morre localmente mas não morre globalmente.

Com argumentos de acoplamento, como os usados na prova da Proposição 2.2.1, podemos construir duas cópias $\eta_{t}$ e $\hat{\eta}_{t}$ de $C C(\mathcal{G}, \lambda, p)$ tal que $\eta_{t} \leq \hat{\eta}_{t}$ para todos os tempos $t>0$, assumindo que $\eta_{0} \leq \hat{\eta}_{0}$. Devido a esta propriedade de monotonicidade (conhecida também como atratividade), com argumentos de dominação, questões de sobrevivência ou extinção são menos difíceis de se tratar. Agora, a fim de analisar o modelo $C C(\mathcal{G}, \lambda, p)$ em relação aos seus parâmetros, definimos

$$
\lambda_{c}(p, \mathcal{G}):=\inf \left\{\lambda: \mathbb{P}^{\delta_{x}}\left(\left|\eta_{t}\right| \geq 1 \text { para todo } t \geq 0\right)>0\right\}
$$

onde $x$ é um vértice fixado, e $\mathbb{P}^{\delta_{x}}$ é a lei do processo $\eta_{t}$ começando com uma única colônia em $x$. Observe que $\lambda_{c}(p, \mathcal{G})$ é uma função não crescente em $p$. Além disso, $\lambda_{c}(1, \mathcal{G})=0$ e $\lambda_{c}(0, \mathcal{G})=\infty$. 
Definição 2.2.3. Seja $\eta_{t}$ um processo $C C(\mathcal{G}, \lambda, p)$ com $0<p<1$. Dizemos que $\eta_{t}$ exibe transição de fase $(\mathrm{em} \lambda)$ se $0<\lambda_{c}(p, \mathcal{G})<\infty$.

Observe que o número de indivíduos por vértice não é limitado. Assim, é concebível pensar que o modelo sobreviva em um grafo finito. No seguinte resultado mostramos que isso não acontece e portanto não existe transição de fase do modelo em grafos finitos.

Proposição 2.2.2. Para $p<1$ e todo grafo finito $\mathcal{G}$, o processo de colonização e colapso $C C(\mathcal{G}, \lambda, p)$ morre.

Demonstração. Seja $\alpha$ a probabilidade de que após o colapso de uma colônia, nenhum de seus indivíduos tente fazer uma nova colônia nos vértices vizinhos. Do fato que um processo de Yule, começando com um indivíduo, tem $j$ indivíduos no tempo $t$ com probabilidade $e^{-\lambda t}\left(1-e^{-\lambda t}\right)^{j-1}$, segue que

$$
\alpha=\int_{0}^{\infty} e^{-t} \sum_{j=1}^{\infty} e^{-\lambda t}\left(1-e^{-\lambda t}\right)^{j-1}(1-p)^{j} d t .
$$

Seja $n$ o número de vértices de $\mathcal{G}$ e $m$ o grau máximo desses vértices. A fim de mostrar condições suficientes para extinção, acoplamos o número de colônias no processo $C C(\mathcal{G}, \lambda, p)$ a um processo de ramificação em tempo contínuo. Seja $X_{t}$ o seguinte processo de ramificação. Cada indivíduo está independentemente associado a uma variável aleatória exponencial de taxa 1. Quando esse tempo exponencial ocorre, o indivíduo morre com probabilidade $\alpha$ ou é substituído por $m$ indivíduos com probabilidade $1-\alpha$. Nós adicionamos a restrição de que o número total de indivíduos no processo é sempre menor ou igual que $n$. Isto é, suprimimos nascimentos que fariam $X_{t}$ maior que este valor.

Seja $C_{t}$ o número de colônias no tempo $t$ no processo de colonização e colapso. No tempo $t=0$ seja $X_{0}=C_{0}$. Além disso, acoplamos cada indivíduo em $X_{0}$ a uma colônia no processo de colonização e colapso usando a mesma variável aleatória exponencial de taxa 1. Quando uma exponencial ocorre existem duas possibilidades. Com probabilidade $\alpha$ ambos $X_{t}$ e $C_{t}$ decrescem uma unidade, ou com probabilidade $1-\alpha$ o processo $X_{t}$ aumenta em $m-1$ indivíduos e $C_{t}$ aumenta em no máximo $m-1$ colônias. Isto porque no processo de colonização temos restrições espaciais e as tentativas de colonizações só são bem sucedidas em vértices que estão vazios. Assim, novas colônias correspondem a nascimentos para $X_{t}$. Acoplamos cada nova colônia a um novo indivíduo em $X_{t}$, usando a mesma variável aleatória exponencial de taxa 1 . 
Com este acoplamento temos que para todo $t \geq 0$

$$
X_{t} \geq C_{t}
$$

Observe agora que $\alpha>0$ e que $X_{t}$ é um processo de Markov com espaço de estados finito e um estado absorvente. Assim, $X_{t}$ morre com probabilidade 1. Portanto, o processo de colonização e colapso morre quase certamente.

\subsection{Resultados principais}

No seguinte teorema mostramos condições suficientes para extinção (global e local) no modelo de colonização e colapso sobre grafos infinitos.

Teorema 2.3.1. Seja $\mathcal{G}$ um grafo m-regular, $\eta_{t}$ um processo $C C(\mathcal{G}, \lambda, p)$ e

$$
\mu(m)=m-\frac{m}{\lambda} \sum_{k \geq 1} B\left(1+\frac{1}{\lambda}, k\right)\left(1-\frac{p}{m}\right)^{k}
$$

onde $B(a, b)=\int_{0}^{1} u^{a-1}(1-u)^{b-1} d u$ é a função Beta.

(i) Se $\mu(m) \leq 1$ e $\left|\eta_{0}\right|<\infty$, então $\eta_{t}$ morre local e globalmente.

(ii) Seja $\mathcal{G}=\mathbb{Z}^{d}, d \geq 1$. Se $\mu(2 d)<1$ e $\eta_{0}(x) \leq 1$ para todo $x$ em $\mathbb{Z}^{d}$ então $\eta_{t}$ morre localmente.

(iii) Seja $\mathcal{G}=\mathbb{T}^{d}, d \geq 1$. Se $\mu(d+1)<1 / d$ e $\eta_{0}(x) \leq 1$ para cada $x$ em $\mathbb{T}^{d}$ então $\eta_{t}$ morre localmente.

Observação 2.3.1. Observe que para todo $m \geq 1$ e $\lambda>0$ fixados, existe $p>0$ tal que $\mu(m) \leq 1$. Além disso, $\mu(m)$ pode ser escrito em termos da função hipergeométrica de Gauss ${ }_{2} F_{1}$ (Ver Luke [17]),

$$
\mu(m)=m-\frac{m-p}{\lambda+1}{ }_{2} F_{1}\left(1,1 ; 2+\frac{1}{\lambda} ; 1-\frac{p}{m}\right)
$$

No seguinte resultado mostramos condições suficientes para a sobrevivência do processo de colonização e colapso sobre alguns grafos infinitos.

Teorema 2.3.2. Para $p>0$ e $\lambda:=\lambda(p, \mathcal{G})>0$ suficientemente grande, o processo $C C(\mathcal{G}, \lambda, p) \operatorname{com} \mathcal{G}=\mathbb{Z}^{d}$ ou $\mathbb{T}^{d}$ sobrevive global e localmente.

As provas dos Teoremas 2.3.1 e 2.3.2 são apresentadas na Seção 2.5. 
Observação 2.3.2. Dos Teoremas 2.3.1 e 2.3.2 segue que para $\mathcal{G}=\mathbb{Z}^{d}$ ou $\mathbb{T}^{d}$, existe transição de fase $(\operatorname{em~} \lambda)$ para o modelo $C C(\mathcal{G}, \lambda, p)$. Isto é, existe uma função $\lambda_{c}(\cdot, \mathcal{G}):(0,1) \rightarrow \mathbb{R}^{+}$tal que a sobrevivência e extinção de $C C(\mathcal{G}, \lambda, p)$ pode ser representada como na Figura 2.1.

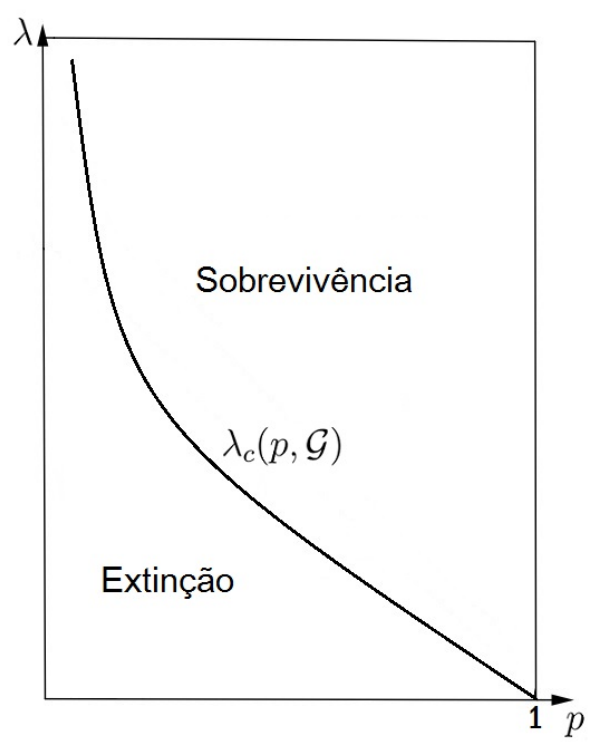

Figura 2.1: Transição de fase para $C C(\mathcal{G}, \lambda, p)$, com $\mathcal{G}=\mathbb{Z}^{d}$ ou $\mathbb{T}^{d}$.

Observação 2.3.3. Sabemos que $\lambda_{c}(p, \mathcal{G})$ é não crescente em $p$. Mas até agora não sabemos se há continuidade e monotonicidade estrita $(\operatorname{em} p)$ da função $\lambda_{c}(p, \mathcal{G})$. Se a monotonicidade é estrita, então o processo também tem transição de fase em $p$ para cada $\lambda \in(0, \infty)$ fixo.

\subsection{Modelos não espaciais}

Modelos não espaciais, também conhecidos como modelos catastróficos, têm sido extensivamente estudados e são muito próximos ao nosso modelo; para referências ao respeito ver Kapodistria et al. [14]. Particularmente relevante é o processo de nascimento e morte com catástrofes, descrito no exemplo 2 em Brockwell [7].

Brockwell [7] considera um modelo não espacial com uma única colônia. Cada indivíduo dá nascimentos a taxa $\lambda>0$ e morre a taxa $\mu>0$. Além disso, catástrofes (colapsos) ocorrem a taxa $a>0$. Quando uma catástrofe ocorre todo indivíduo na colônia tem probabilidade $p$ de sobreviver e $1-p$ de morrer, independente do que ocorre a outros indivíduos. Brockwell [7] mostrou que a sobrevivência (isto é, em todos os 
instantes de tempo existe pelo menos um indivíduo na colônia) tem probabilidade positiva se e somente se $\lambda>\mu$ e

$$
\mu-a \log p<\lambda
$$

Portanto, existe um valor crítico para $p$

$$
p_{1}=\exp \left(-\frac{\lambda-\mu}{a}\right)
$$

O modelo com uma única colônia sobrevive se e somente se $p>p_{1}$.

Agora nós introduzimos uma versão não espacial do nosso modelo e comparamos este com o modelo de catástrofe descrito anteriormente. Considere um modelo para o qual todo indivíduo dá nascimentos a taxa $\lambda$ e morre a taxa $\mu$. Nós iniciamos o processo com um único indivíduo e portanto com uma única colônia. Quando uma colônia colapsa seus indivíduos sobrevivem com probabilidade $p$ e morrem com probabilidade $1-p$, independentemente do que ocorre com outros indivíduos. Todo indivíduo sobrevivente gera uma nova colônia a qual eventualmente colapsa. Colônias colapsam independentemente de outras a taxa $a>0$. A prova do Teorema 1.2.1 (Teorema 1 em [20]) pode ser adaptada para mostrar a seguinte propriedade deste modelo de múltiplas colônias.

Proposição 2.4.1. O modelo de múltiplas colônias sobrevive com probabilidade positiva se e somente se

$$
p \mathbb{E}[\exp ((\lambda-\mu) T)]>1
$$

onde $T$ tem distribuição exponencial com taxa a.

Demonstração. Assumimos que o processo começa com uma única colônia e definimos $V_{0}=1$. Após o colapso da colônia, seus sobreviventes se dispersam gerando um número aleatório $V_{1}$ (possivelmente 0 ) de novas colônias. Cada uma das $V_{1}$ colônias sofre um colapso em diferentes tempos aleatórios e cada sobrevivente gera uma nova colônia. Denotemos por $V_{2}$ o número total dessas novas colônias, e assim por diante. O processo resultante, $\left(V_{n}\right)_{n \geq 0}$, é um processo Galton-Watson, o qual sobrevive se e somente se $\mathbb{E}\left[V_{1}\right]>1$. Observe que o modelo de múltiplas colônias sobrevive se e somente se o processo $\left(V_{n}\right)_{n \geq 0}$ sobrevive.

A fim de calcular $\mathbb{E}\left[V_{1}\right]$, definimos $Y$ como o número de indivíduos na primeira colônia no tempo do colapso. Observe que a variável $V_{1}$ condicionada ao evento 
$\{Y=k\}$, tem distribuição Binomial de parâmetros $(k, p)$. Portanto,

$$
\mathbb{E}\left[V_{1}\right]=\mathbb{E}\left[\mathbb{E}\left[V_{1} \mid Y\right]\right]=\mathbb{E}[p Y]=p \mathbb{E}[Y]
$$

Seja $T$ o tempo de colapso da colônia. Usando o fato de que o número esperado de indivíduos no tempo $t$ em um processo de nascimento e morte, começando com um indivíduo, é $\exp ((\lambda-\mu) t)$, temos que

$$
\mathbb{E}[Y]=\mathbb{E}[\exp (\lambda-\mu) T]
$$

Disto segue o resultado.

É fácil ver que se $\lambda \geq \mu+a$ então o valor esperado do lado esquerdo da desigualdade da Proposição 2.4.1 é $+\infty$ e a desigualdade vale para todo $p>0$. Pode-se observar também que a desigualdade não vale se $\lambda \leq \mu$. Logo, de agora em diante vamos assumir que $\mu<\lambda<\mu+a$. Após calcular o valor esperado e resolver a desigualdade em $p$ nós concluímos que o evento sobrevivência tem probabilidade positiva se e somente se

$$
p>1-\frac{\lambda-\mu}{a}
$$

Isto é, quando $\mu<\lambda<\mu+a$ o modelo com várias colônias tem um valor crítico

$$
p_{2}=1-\frac{\lambda-\mu}{a}
$$

O modelo com múltiplas colônias sobrevive se e somente se $p>p_{2}$.

Como $\exp (-x)>1-x$ para todo $x \neq 0$ nós temos que $p_{1}>p_{2}$ para todo $\lambda>\mu$. Logo, é mais provável para o modelo com múltiplas colônias sobreviver do que o modelo com uma única colônia. Isto é, morar em diversas colônias pequenas é uma estratégia melhor do que morar em uma única grande colônia. Note que esta conclusão não é óbvia. O modelo com uma única colônia tem taxa de catástrofe $a$ enquanto o modelo com múltiplas colônias tem taxa de catástrofe $n a$ se existem $n$ colônias. Além disso, uma catástrofe tem maior probabilidade de acabar com uma colônia menor do que com uma maior. Por outro lado, múltiplas colônias dão várias possibilidades para sobrevivência e isso acaba sendo uma importante vantagem do modelo com múltiplas colônias em relação ao modelo com uma única colônia. 


\subsection{Provas}

Apresentamos nesta seção as provas dos Teoremas 2.3.1 e 2.3.2. Precisamos para isso de alguns resultados auxiliares.

\subsubsection{Resultados auxiliares}

Seja $\eta_{t}$ um processo $C C(\mathcal{G}, \lambda, p)$. Note que neste processo, algumas tentativas de colonização não são bem sucedidas porque o vértice no qual ocorre a tentativa já está ocupado. Assim, quando ocorre um colapso em uma colônia o número de novas colônias geradas pelos indivíduos sobreviventes depende do número de vértices vizinhos vazios. Isto gera dependência entre o número de novas colônias criadas após o colapso de diferentes colônias. Por esta falta de independência, torna-se inviável o cálculo explícito de probabilidades. A fim de provar o Teorema 2.3.1, nós introduzimos um processo de ramificação que domina, em certo sentido, o processo $\eta_{t}$ e para o qual os cálculos explícitos de probabilidades são possíveis. Este processo será denotado por $\xi_{t}$ e é descrito a seguir.

\section{Processo auxiliar $\xi_{t}$ :}

No processo $\xi_{t}$ cada vértice de $\mathcal{G}$ pode estar vazio ou ocupado por um número finito (não limitado) de colônias. Cada colônia inicia com um único indivíduo. O número de indivíduos em uma colônia no tempo $t$ é determinado por um processo de nascimento puro de taxa $\lambda>0$. Cada colônia está associada a uma variável aleatória (tempo) exponencial de média 1. Quando o tempo exponencial ocorre, a colônia colapsa e independente de qualquer coisa, cada indivíduo sobrevive com probabilidade $p>0$ ou morre com probabilidade $1-p$. Cada indivíduo que sobrevive tenta criar uma nova colônia em um dos vértices vizinhos escolhido de forma aleatória. Se dois ou mais indivíduos provenientes de uma mesma colônia colapsada tentam criar colônia no mesmo vértice, apenas uma nova colônia é criada. Logo, no processo $\xi_{t}$ quando uma colônia localizada no vértice $x$ colapsa, ela é substituída por $0,1, .$. ou grau $(x)$ novas colônias, estando cada nova colônia sobre um vértice vizinho do vértice $x$.

Observe que as taxas de nascimento e colapso são as mesmas para colônias em $\xi_{t}$ e $\eta_{t}$. Cada nova colônia criada no processo $\eta_{t}$ corresponde a uma colônia criada no processo $\xi_{t}$. Porém, nem toda colônia criada no processo $\xi_{t}$ tem sua correspondente no processo $\eta_{t}$. Técnicas como as usadas em Liggett [16, Teorema 1.5 no capítulo III] podem ser usadas para construir os processos $\xi_{t}$ e $\eta_{t}$ em um mesmo espaço de probabilidade de modo que se eles iniciam com uma mesma configuração, se existir 
uma colônia de tamanho $i$ em um vértice $x$ para $\eta_{t}$ então existirá pelo menos uma colônia de tamanho $i$ para $\xi_{t}$ no mesmo vértice $x$.

Lema 2.5.1. Seja $\mathcal{G}$ um grafo $m$-regular e $W_{m}(\lambda, p)$ o número de novas colônias criadas por indivíduos de uma colônia que colapsa no processo $\xi_{t}$ em $\mathcal{G}$. Então

(i) $\mu(m):=\mathbb{E}\left[W_{m}(\lambda, p)\right]=m-\frac{m}{\lambda} \sum_{k \geq 1} B\left(1+\frac{1}{\lambda}, k\right)\left(1-\frac{p}{m}\right)^{k}$.

(ii) $q_{\lambda}:=\mathbb{P}\left[W_{m}(\lambda, p)=m\right] \rightarrow 1$ quando $\lambda \rightarrow \infty$.

Observação 2.5.1. Observe que para o processo $\eta_{t}$ a probabilidade de que após o colapso no vértice $x$ cada um dos seus $m$ vizinhos recebam pelo menos uma tentativa de colonização é igual a $q_{\lambda}$.

Prova do Lema 2.5.1 $(i)$. Considere uma colônia em algum vértice $x$ de $\mathcal{G}$. Seja $Y$ o número de indivíduos na colônia no tempo de colapso. Então, por condicionamento no tempo do colapso,

$$
\mathbb{P}[Y=k]=\int_{0}^{\infty} e^{-t} e^{-\lambda t}\left(1-e^{-\lambda t}\right)^{k-1} d t=\frac{1}{\lambda} B\left(1+\frac{1}{\lambda}, k\right)
$$

onde a última igualdade é obtida pela substituição $u=e^{-\lambda t}$ e da definição da função beta.

Enumere cada vizinho do vértice $x$ de 1 a $m$. A seguir, escreva $W_{m}(\lambda, p)=\sum_{i=1}^{m} I_{i}$, onde $I_{i}$ é a função indicadora do evento \{Uma nova colônia é criada no $i$-ésimo vizinho de $x\}$. Logo,

$$
\mathbb{E}\left[W_{m}(\lambda, p)\right]=\sum_{i=1}^{m} \mathbb{P}\left[I_{i}=1\right]=m \mathbb{P}\left[I_{1}=1\right]
$$

Observe que

$$
\mathbb{P}\left[I_{1}=1 \mid Y=k\right]=1-\left(1-\frac{p}{m}\right)^{k}
$$

Logo,

$$
\begin{aligned}
\mathbb{P}\left[I_{1}=1\right] & =\sum_{k=1}^{\infty}\left[1-\left(1-\frac{p}{m}\right)^{k}\right] \mathbb{P}[Y=k] \\
& =1-\frac{1}{\lambda} \sum_{k=1}^{\infty}\left(1-\frac{p}{m}\right)^{k} B\left(1+\frac{1}{\lambda}, k\right)
\end{aligned}
$$


onde a última desigualdade é obtida por (2.5.1). Substituindo (2.5.3) em (2.5.2) nós obtemos o resultado desejado.

Prova do Lema 2.5.1 (ii). Observe que

$$
\begin{aligned}
\mathbb{P}\left[W_{m}(\lambda, p)=m\right] & =\mathbb{P}\left[I_{1}=1, \ldots, I_{m}=1\right] \\
& =1-\mathbb{P}\left[I_{i}=0 \text { para algum } i \in 1, \ldots m\right] \\
& \geq 1-\sum_{i=1}^{m} \mathbb{P}\left[I_{i}=0\right] \\
& =1-m \mathbb{P}\left[I_{1}=0\right] \\
& =1-\frac{m}{\lambda} \sum_{k=1}^{\infty}\left(1-\frac{p}{m}\right)^{k} B\left(1+\frac{1}{\lambda}, k\right) \\
& \geq 1-\frac{m}{\lambda} \sum_{k=1}^{\infty}\left(1-\frac{p}{m}\right)^{k} B(1, k)
\end{aligned}
$$

Fazendo $\lambda \rightarrow \infty$ em (2.5.4) nós obtemos o resultado desejado.

\subsubsection{Provas dos resultados principais}

Prova do Teorema 2.3.1 (i). Considere $\xi_{t}$ iniciando com uma única colônia em um vértice de $\mathcal{G}$ e definamos $Z_{0}=1$. Chamemos esta colônia de geração 0. Após o colapso desta colônia, um número aleatório de novas colônias são criadas. Denotemos este número aleatório por $Z_{1}$. Estas formam a primeira geração de colônias. Cada colônia da primeira geração dá nascimentos (em tempos aleatórios diferentes) a um número aleatório de novas colônias. Estas novas colônias formarão a segunda geração de colônias e sua quantidade é denotada por $Z_{2}$. Em geral, para $n \geq 1$, se $Z_{n-1}=0$ então $Z_{n}=0$, se $Z_{n-1} \geq 1$ então $Z_{n}$ é o número de colônias geradas pelas colônias da geração $n-1$.

Nós afirmamos que $Z_{n}, n=0,1, \ldots$ é um processo de ramificação Galton-Watson. Isto é verdade já que os números de descendentes de diferentes colônias no processo $\xi_{t}$ têm a mesma distribuição e são independentes.

O processo $Z_{n}$ morre quase certamente se e somente se $\mathbb{E}\left[Z_{1}\right] \leq 1$. Do Lema 2.5.1.( $i$ ) nós sabemos que $\mathbb{E}\left[Z_{1}\right]=\mu(m)$.

Observe que se o processo $Z_{n}$ morre quase certamente, o mesmo ocorre para $\xi_{t}$ e $\operatorname{logo}$ para $\eta_{t}$. É fácil ver que esta prova pode ser adaptada para o caso onde o processo se inicia com qualquer quantidade finita de colônias.

Prova do Teorema 2.3.1 (ii). Da propriedade de monotonicidade de $\eta_{t}$ é suficiente 
mostrar extinção local para o processo iniciando com um único indivíduo em cada vértice de $\mathbb{Z}^{d}$. Provaremos esta afirmação para o processo $\xi_{t}$ iniciando com uma colônia em cada sítio de $\mathbb{Z}^{d}$.

Fixe um vértice $x$. Se no tempo $t$ existe uma colônia no vértice $x$ (para o processo $\xi_{t}$ ) então ela deve descender de uma colônia presente no tempo 0. Assuma que a colônia em $x$ descende de uma colônia no vértice $y$. Seja $Z_{n}(y)$ o número de colônias na $n$-ésima geração da colônia que iniciou em $y$. O processo $Z_{n}(y)$ tem a mesma distribuição que o processo $Z_{n}$ definido anteriormente. Para que um descendente de $y$ eventualmente alcance $x$ o processo $Z_{n}(y)$ deve ter sobrevivido pelo menos $d(x, y)$ gerações. Isto ocorre porque cada geração somente dá nascimentos em seus vértices vizinhos mais próximos. O processo $Z_{n}(y)$ é um processo de ramificação GaltonWatson com $Z_{0}(y)=1$ e número médio de descendentes $\mu=\mu(2 d)$.

Seja $n=d(x, y)$, então

$$
\mathbb{P}\left(Z_{n}(y) \geq 1\right) \leq \mathbb{E}\left(Z_{n}(y)\right)=\mu^{n}=\mu^{d(x, y)}
$$

e

$$
\sum_{y \in \mathbb{Z}^{d}} \mathbb{P}\left(Z_{n}(y) \geq 1\right) \leq \sum_{y \in \mathbb{Z}^{d}} \mu^{d(x, y)}<\infty
$$

para $\mu<1$.

O Lema de Borel-Cantelli mostra que quase certamente existe apenas um número finito de $y$ 's tais que descendentes de $y$ eventualmente alcançam $x$. De $(i)$ nós sabemos que se o processo inicia com um número finito de indivíduos ele morre quase certamente. Logo, após um tempo aleatório finito não irá existir nenhuma colônia no vértice $x$.

Prova do Teorema 2.3.1 (iii). A prova é análoga a (ii). Neste caso, $\mu=\mu(d+1)$ e

$$
\sum_{y \in \mathbb{T}^{d}} \mu^{d(x, y)}<\infty \Longleftrightarrow \mu<1 / d
$$

Prova do Teorema 2.3.2: Nós primeiro provamos o resultado para a rede unidimensional $\mathbb{Z}$. Nesta prova usaremos a construção de blocos apresentada em Bramson e Durrett [8]. Primeiro apresentamos algumas notações. Para $m$ e $n$ inteiros nós definimos

$$
I=[-L, L] \quad I_{m}=2 m L+I
$$




$$
B=(-4 L, 4 L) \times[0, T] \quad B_{m, n}=(2 m L, n T)+B,
$$

onde

$$
T=\frac{5}{2} L
$$

$\mathrm{e}$

$$
\mathbb{L}=\left\{(m, n) \in \mathbb{Z}^{2}: m+n \text { é par }\right\} .
$$

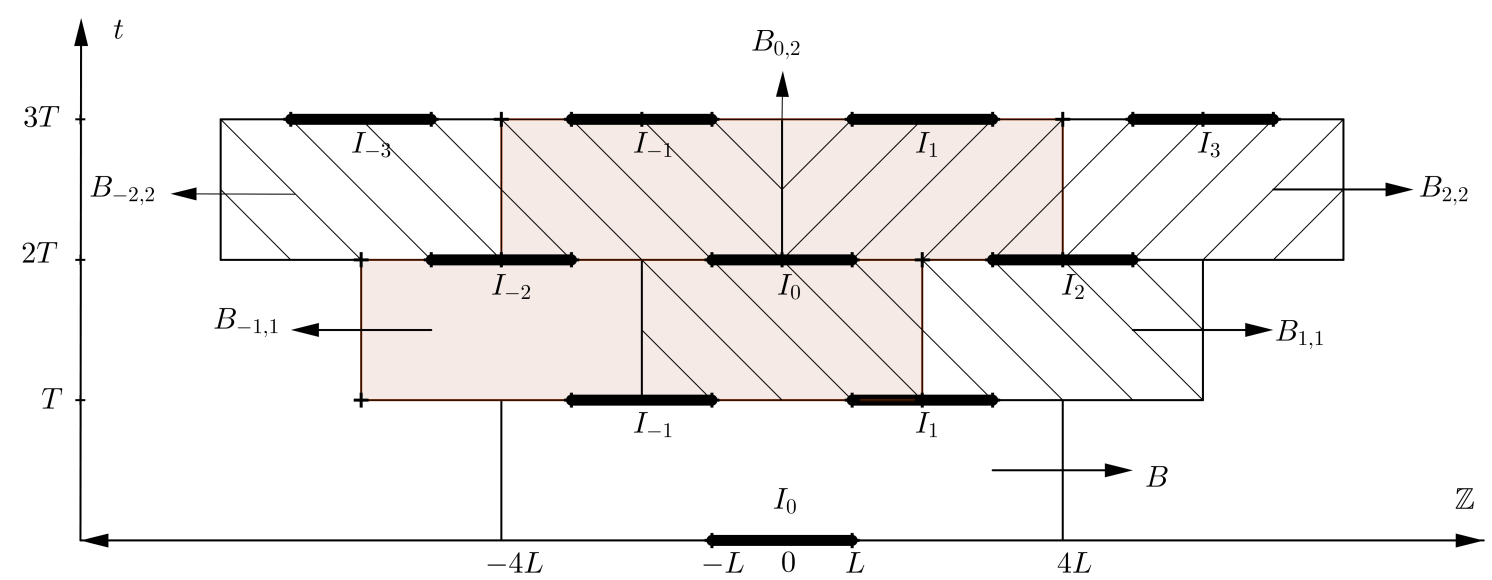

Figura 2.2: Modelo de colonização e colapso em $\mathbb{Z}$.

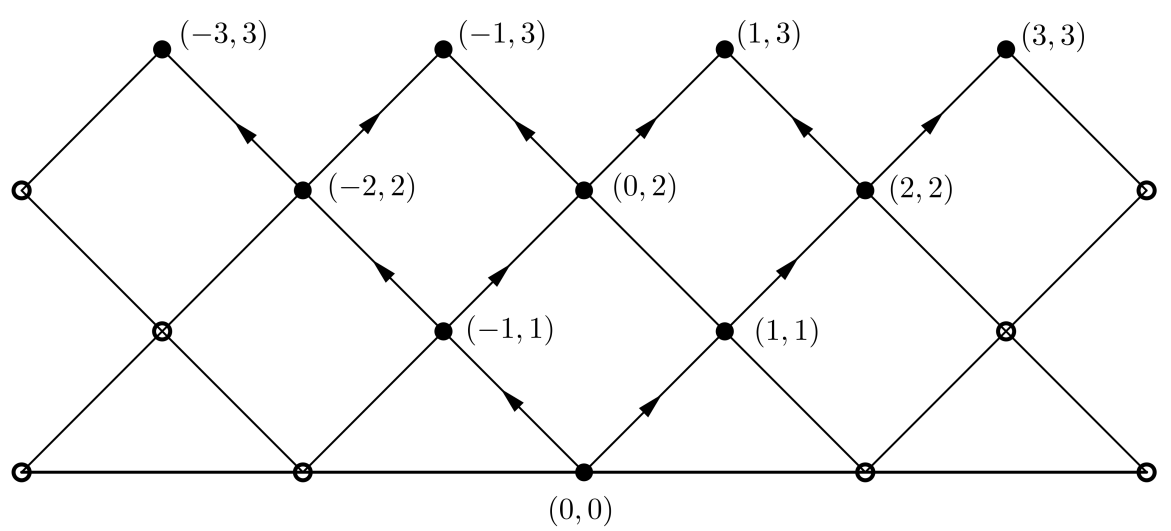

Figura 2.3: Modelo de percolação orientada em $\mathbb{L}$.

Nós dizemos que o intervalo $I_{m}$ é quase cheio se em todo par de vértices vizinhos em $I_{m}$ há pelo menos um vértice ocupado. Isto é, o número máximo de vértices consecutivos desocupados em $I_{m}$ é no máximo 1 .

Nós dizemos que $(m, n) \in \mathbb{L}$ é molhado se iniciando com $I_{m}$ quase cheio no tempo $n T$ então $I_{m-1}$ e $I_{m+1}$ são também quase cheios no tempo $(n+1) T$. Além disso, nós 
queremos que o último evento aconteça somente usando os processos de Yule e Poisson dentro da caixa $B_{m, n}$. Isto é, nós consideramos o processo restrito a $B_{m, n}$.

Agora, vamos mostrar que para qualquer $\epsilon>0$ existem $\lambda, L$ e $T$ tal que para todo $(m, n) \in \mathbb{L}$

$$
P((m, n) \text { é molhado }) \geq 1-\epsilon .
$$

Pela invariância por translação é suficiente provar para $(m, n)=(0,0)$. A prova tem dois passos.

- Seja $E$ o evento que todo colapso na caixa finita espaço-temporal $B$ é seguido de pelo menos uma tentativa de colonização à esquerda e outra à direita do vértice que colapsa. Nós afirmamos que para cada $\epsilon>0$, podemos escolher $L, T$ e $\lambda>0$ suficientemente grandes tais que $P(E) \geq 1-\epsilon$. Vamos agora argumentar por que isso é verdade. Como os tempos de colapso em cada vértice de $B$ são dados por um processo Poisson de taxa 1, o número total de colapsos dentro de $B$ é limitado por cima por uma distribuição Poisson de taxa $(8 L+1) T$. Assim, com alta probabilidade existem menos que $2(8 L+1) T$ colapsos dentro de $B$ para $L$ suficientemente grande. Também tomamos $\lambda$ suficientemente grande de modo que em cada tempo de colapso a colônia terá muitos indivíduos que tentaram colonizações à esquerda e à direita com alta probabilidade (ver Lema 2.5.1.(ii)). Dado que o número de colapsos pode ser limitado com alta probabilidade, a probabilidade do evento $E$ pode ser feita arbitrariamente próxima a 1.

- No tempo 0 nós começamos o processo com o intervalo $I$ quase cheio. Sejam $r_{t}$ e $\ell_{t}$ os vértices ocupados mais à direita e mais à esquerda, respectivamente, no tempo $t \geq 0$. Condicionado ao evento $E$, é fácil ver que o intervalo $\left[\ell_{t}, r_{t}\right]$ é quase cheio em qualquer tempo $t \leq T$. Observe também que condicionado a $E$, cada vez que existe um colapso em $r_{t}$, então $r_{t}$ pula para $r_{t}+1$. Dado que o número de colapsos em $r_{t}$ é um processo de Poisson com taxa 1, nós temos que $\frac{r_{t}}{t}$ converge para 1. Assim, para $T=\frac{5}{2} L$ e $L$ suficientemente grande, temos que $r_{T}$ pertence a $(3 L, 4 L)$ com uma probabilidade arbitrariamente próxima de 1 . Um argumento análogo mostra que $\ell_{T}$ pertence a $(-4 L,-3 L)$. Já que o intervalo $\left[\ell_{T}, r_{T}\right]$ contem a $I_{-1}$ e $I_{1}$, ambos estes intervalos são quase cheios. Assim, para qualquer $\epsilon>0$ podemos escolher $L$ e $\lambda$ suficientemente grandes de modo que $P((0,0)$ é molhado $) \geq 1-\epsilon$.

A construção anterior dá um acoplamento entre nosso modelo de colonização e colapso e um modelo de percolação orientada em $\mathbb{L}$; veja as Figuras 2.2 e 2.3. O modelo de percolação orientada é 1-dependente e é bem conhecido que para $\epsilon>0$ suficientemente pequeno, $(0,0)$ estará em um aglomerado infinto de percolação que contém infinitos vértices do tipo $(0,2 n)$, ver Durrett [9]. Este fato corresponde, pelo acoplamento, à sobrevivência local no modelo de colonização e colapso. Note que a 
prova foi feita para o processo restrito às caixas $B_{m, n}$. Se este processo sobrevive localmente, então o mesmo acontece para o modelo não restrito. Isto devido ao fato de que o modelo é atrativo e mais nascimentos só podem ajudar a sobrevivência.

Consideremos agora $\mathbb{Z}^{d}$ com $d \geq 2$. Primeiro observe que do caso $d=1$, nós temos uma condição suficiente para sobrevivência local para o processo restrito a uma linha fixada de $\mathbb{Z}^{d}$. Uma vez que o processo é atrativo, tal condição é suficiente para mostrar sobrevivência local em $\mathbb{Z}^{d}$. Isto é assim porque o modelo irrestrito trará mais nascimentos (mas não mais mortes) para os vértices na linha fixada.

Para $\mathbb{T}^{d}$ a sobrevivência local segue analogamente como em $\mathbb{Z}^{d}$, observando que $\mathbb{Z}$ está imerso em $\mathbb{T}^{d}$. 


\section{Capítulo 3}

\section{Dispersão como uma estratégia de sobrevivência}

\subsection{Introdução}

Em Artalejo et al. [1] foi considerado um modelo para crescimento de uma população (uma única colônia) sujeita a colapsos. Dois tipos de colapsos foram analisados separadamente, catástrofes binomiais e catástrofes geométricas. Após os colapsos, os sobreviventes continuam juntos na mesma colônia (isto é, não existe dispersão). Com base nesse modelo e seguindo as ideias do Capítulo 2, neste capítulo introduzimos novos modelos de crescimento de populações sujeitas a colapsos. ${ }^{1}$

Este capítulo está dividido em quatro seções. Na Seção 2 definimos e caracterizamos três modelos para crescimento de populações sujeitas a colapsos. Na Seção 3 comparamos os três modelos introduzidos na Seção 2 e determinamos em que condições a dispersão é uma boa estratégia de sobrevivência, em função das restrições espaciais e dos tipos de colapsos. Finalmente, na Seção 4 provamos os resultados estabelecidos nas Seções 2 e 3.

\subsection{Modelos de crescimento}

Descrevemos primeiro o modelo de Artalejo et al. [1]. Este é um modelo de uma única colônia. A colônia dá nascimento a um novo indivíduo a taxa $\lambda>0$, e colapsos ocorrem a taxa $\mu$. Se na ocorrência de um colapso o tamanho da população é $i$, esta se reduz a um tamanho $j$ com probabilidade $\mu_{i j}$. O valor $\mu_{i j}$ é determinado pelo tipo

\footnotetext{
${ }^{1}$ Os resultados deste Capítulo estão reunidos em Junior et al. [12]
} 
de colapso. São considerados dois tipos de colapsos descritos a seguir.

- Catástrofe Binomial: Os indivíduos são expostos ao efeito catastrófico simultaneamente. Cada indivíduo sobrevive com probabilidade $p<1$ (morre com probabilidade $q=1-p)$, independentemente de qualquer outra coisa. Isto é,

$$
\mu_{i j}^{B}=\left(\begin{array}{l}
i \\
j
\end{array}\right) p^{i} q^{i-j}, 0 \leq j \leq i .
$$

- Catástrofe Geométrica: Os indivíduos são expostos ao efeito catastrófico sequencialmente e o declínio na população para no primeiro indivíduo que sobrevive, ou quando toda a população torna-se extinta. Se cada indivíduo sobrevive com probabilidade $p$, então

$$
\mu_{i j}^{G}= \begin{cases}q^{i}, & j=0 \\ p q^{i-j}, & 1 \leq j \leq i .\end{cases}
$$

\subsubsection{Modelo sem dispersão}

Em Artalejo et al. [1] os autores consideram os modelos com catástrofe binomial e catástrofe geométrica de forma isolada. Em outras palavras, apresentam uma versão do processo para cada tipo de catástrofe. Dado que é considerável pensar que diferentes tipos de colapsos podem afetar uma mesma população, vamos considerar aqui um modelo que mistura os dois tipos (catástrofes binomiais e geométricas). Isto é, nos tempos de colapso, com probabilidade $r$ o colapso é do tipo catástrofe geométrica e com probabilidade $1-r$ o colapso é do tipo catástrofe binomial. Assim,

$$
\mu_{i j}:=r \mu_{i j}^{G}+(1-r) \mu_{i j}^{B}
$$

Assumimos também que $\mu=1$. Mais formalmente, o tamanho da população (número de indivíduos na colônia) no tempo $t$ é um processo de Markov a tempo contínuo $\{X(t): t \geq 0\}$ com gerador infinitesimal $\left(q_{i j}\right)_{i, j \geq 0}$ dado por

$$
q_{i j}= \begin{cases}\lambda, & j=i+1, i \geq 0 \\ \mu_{i j}, & 0 \leq j<i \\ -\left(\lambda+\sum_{j=0}^{i-1} \mu_{i j}\right), & i=j \\ 0 & \text { em outro caso. }\end{cases}
$$

Assumindo $X(0)=1$, denotaremos por $M C^{1}(r, \lambda, p)$ o modelo descrito pelo processo $\{X(t): t \geq 0\}$. Fazendo $r=0$ e $r=1$, obtemos os modelos considerados em Artalejo et al. [1]. 
Teorema 3.2.1 (Artalejo et al. [1]). Seja $X(t)$ um processo $M C^{1}(r, \lambda, p)$, com $\lambda>0$ $e 0<p<1$. Então, a extinção (isto é, $X(t)=0$ para algum $t>0$ ) ocorre com probabilidade

$$
\rho_{1}(r)= \begin{cases}1 & , \text { se } r<1 \\ \min \left\{\frac{1-p}{\lambda p}, 1\right\} & , \text { se } r=1 .\end{cases}
$$

Além disso, se $r<1$, ou $r=1$ e $\lambda p<1-p$, o tempo até a extinção do processo tem média finita.

Observação 3.2.1. O resultado do Teorema 3.2.1 já tinha sido provado por Artalejo et al. [1] nos casos $r=0$ e $r=1$. A partir do seu resultado pode-se ver que a sobrevivência é possível apenas quando as catástrofes são puramente geométricas $(r=1)$. A razão para isso é bastante clara: se $r<1$ as catástrofes binomiais acontecem a taxa $(1-r)>0$, assim mesmo se considerar $p=1$ quando a catástrofes são geométricas, a população vai morrer como comprovado em Artalejo et al. [1] para o caso $r=0$.

\subsubsection{Modelo com dispersão e sem restrições espaciais}

Considere uma população de indivíduos divididos em colônias independentes. Cada colônia começa com um indivíduo. O número de indivíduos na colônia incrementa segundo um processo de Poisson de taxa $\lambda>0$. De forma independente, a cada colônia é associado um tempo exponencial de média 1. Quando o tempo exponencial ocorre, a colônia colapsa (ocorre uma catástrofe binomial ou geométrica) e cada um dos sobreviventes ao colapso começa uma nova colônia de forma independente. Após o colapso da colônia, ela desaparece. Vamos denotar esta dinâmica por $M C^{2}(r, \lambda, p)$ iniciando com um único indivíduo (uma colônia).

No resultado a seguir estabelecemos condições necessárias e suficientes para a sobrevivência (existência de colônias em todo instante de tempo) no modelo $M C^{2}(r, \lambda, p)$.

Teorema 3.2.2. O modelo $M C^{2}(r, \lambda, p)$ sobrevive com probabilidade positiva se $e$ somente se

$$
\frac{p(\lambda+1)^{2} r}{\lambda p+1}+p(\lambda+1)(1-r)>1
$$

O Teorema 3.2.2 mostra que, ao contrário do que acontece no $M C^{1}(r, \lambda, p)$, em $M C^{2}(r, \lambda, p)$ a população pode sobreviver mesmo quando catástrofes binomiais estão 
presentes $(r<1)$. Veja o exemplo 3.2.1. Em particular, se $r=0$ (só catástrofes binomiais) o processo sobrevive com probabilidade positiva quando $p(\lambda+1)>1$.

No resultado a seguir apresentamos o modo de calcular a probabilidade de extinção, ou seja, a probabilidade de que em algum instante de tempo o sistema fique sem colônias.

Teorema 3.2.3. Seja $\rho_{2}(r)$ a probabilidade de extinção no modelo $M C^{2}(r, \lambda, p)$. Então $\rho_{2}(r)$ é a menor solução não negativa de

$$
\phi(s):=\frac{1}{1+\lambda p}\left[q+\frac{r(\lambda+1) p s}{1+\lambda-\lambda s}+\frac{(1-r)(\lambda+1) p s}{1+\lambda p-\lambda p s}\right]=s
$$

Exemplo 3.2.1. Considere $M C^{2}(r, 1,2 / 5)$. Neste caso,

$$
\phi(s)=\frac{3}{7}+\frac{4 r s}{14-7 s}+\frac{20(1-r) s}{49-14 s} .
$$

Além disso, a menor solução não negativa de $\phi(s)=s$, é dada por

$$
\rho_{2}(r)=\left\{\begin{array}{cc}
1 & , r \leq 7 / 12 \\
\frac{12 r+49-\sqrt{144 r^{2}+1176 r+49}}{28} & , r>7 / 12 .
\end{array}\right.
$$

Observação 3.2.2. Nos casos $r=0$ (só catástrofes binomiais) e $r=1$ (só catástrofes geométricas) a solução de (3.2.2) é simples:

$$
\rho_{2}(0)=\min \left\{\frac{q}{\lambda p}, 1\right\} \quad \text { e } \quad \rho_{2}(1)=\min \left\{\frac{q(\lambda+1)}{\lambda(1+\lambda p)}, 1\right\} .
$$

Observe que $\rho_{2}(0) \geq \rho_{2}(1)$ onde a desigualdade é estrita quando $\left(1+\lambda+\lambda^{2}\right)^{-1}<$ $p<1$. Mais ainda,

- Se $p<\frac{1}{1+\lambda+\lambda^{2}}$ então $\rho_{2}(0)=\rho_{2}(1)=1$.

- Se $\frac{1}{1+\lambda+\lambda^{2}}<p<\frac{1}{1+\lambda}$ então $\rho_{2}(0)=1$ e $\rho_{2}(1)=\frac{q(\lambda+1)}{\lambda(1+\lambda p)}$.

- Se $p>\frac{1}{1+\lambda}$ então $\rho_{2}(0)=\frac{q}{\lambda p}$ e $\rho_{2}(1)=\frac{q(\lambda+1)}{\lambda(1+\lambda p)}$.

Note que, assim como no modelo sem dispersão, a catástrofe binomial é mais severa que a catástrofe geométrica.

Observação 3.2.3. Observe que $\rho_{1}(r) \geq \rho_{2}(r)$. Em particular, se $r<1$ a desigualdade é estrita quando (3.2.1) ocorre. Além disso, $\rho_{1}(1)>\rho_{2}(1)$ para $\lambda(1+\lambda p)>q(\lambda+1)$. 
Portanto, quando não existem restrições espaciais a dispersão é uma boa estratégia para a sobrevivência da população. Isto coincide com os resultados encontrados no modelo de Schinazi [20] e no modelo de colonização e colapso do Capítulo 2.

\subsubsection{Modelo com dispersão e restrições espaciais}

Seja $\mathcal{G}$ um grafo $m$-regular. Cada vértice de $\mathcal{G}$ pode estar vazio ou ocupado por um número finito de colônias. Cada colônia começa com um indivíduo. O número de indivíduos na colônia aumenta segundo um processo de Poisson de taxa $\lambda>0$. Cada colônia é associada a um tempo exponencial de média 1. Quando o tempo exponencial ocorre, a colônia colapsa (ocorre uma catástrofe binomial ou geométrica). Cada um dos sobreviventes ao colapso tenta criar uma nova colônia em um dos vértices vizinhos escolhido aleatoriamente (mesmo que esse vértice já esteja ocupado). Entre os sobreviventes que pulam para um mesmo vértice tentando criar uma nova colônia, somente um tem sucesso; os demais morrem. Assim, neste processo quando uma colônia colapsa, esta é substituída por $0,1, \ldots$ ou $m$ colônias. Denotaremos este modelo por $M C_{m}^{3}(r, \lambda, p)$ e assumimos que o processo começa com um único indivíduo em um vértice de $\mathcal{G}$.

Teorema 3.2.4. O modelo $M C_{m}^{3}(r, \lambda, p)$ sobrevive com probabilidade positiva, se e somente se,

$$
\frac{m p(1+\lambda)^{2} r}{(m+\lambda)(\lambda p+1)}+\frac{m p(1+\lambda)(1-r)}{m+\lambda p}>1
$$

O Teorema 3.2.4 dá uma condição necessária e suficiente para a sobrevivência da população no modelo $M C_{m}^{3}(r, \lambda, p)$. No seguinte resultado mostramos uma forma para calcular a probabilidade de extinção do processo como uma raiz de um polinômio de grau $m$.

Teorema 3.2.5. Seja $\rho_{3}(r)$ a probabilidade de extinção no modelo $M C_{m}^{3}(r, \lambda, p)$. Então $\rho_{3}(r)$ é a menor solução não negativa de

$$
\psi(s):=r \psi_{G}(s)+(1-r) \psi_{B}(s)=s,
$$

onde

$$
\begin{aligned}
& \psi_{B}(s):=\frac{q}{1+\lambda p}+\frac{m(1+\lambda)}{\lambda} \sum_{k=1}^{m}\left(\begin{array}{c}
m \\
k
\end{array}\right)\left[\frac{-\lambda p s}{m(1+\lambda p)}\right]^{k} \sum_{j=0}^{k}\left(\begin{array}{c}
k \\
j
\end{array}\right) \frac{(-1)^{j} j^{k}}{m(1+\lambda p)-\lambda p j} \\
& \psi_{G}(s):=\frac{q}{1+\lambda p}+\frac{(1+\lambda) p s}{\lambda p+1} \sum_{k=1}^{m}\left(\begin{array}{c}
m \\
k
\end{array}\right)\left[\frac{-\lambda s}{m(1+\lambda)}\right]^{k-1} \sum_{j=0}^{k}\left(\begin{array}{c}
k \\
j
\end{array}\right) \frac{(-1)^{j-1} j^{k}}{m(1+\lambda)-\lambda j}
\end{aligned}
$$


Exemplo 3.2.2. Considere $M C_{3}^{3}(r, 1,2 / 3)$. Então

$$
\psi(s)=\left(\frac{126 r}{3575}+\frac{32}{715}\right) s^{3}+\left(\frac{138 r}{3575}+\frac{144}{715}\right) s^{2}+\left(\frac{36}{65}-\frac{24 r}{325}\right) s+\frac{1}{5} .
$$

Logo, a menor solução não negativa de $\psi(s)=s$, é dada por

$$
\rho_{3}(r)=\frac{-440-132 r+\sqrt{22\left(14000+9375 r+792 r^{2}\right)}}{2(80+63 r)} .
$$

\subsection{Dispersão como uma estratégia de sobrevivência}

A fim de avaliar a dispersão como estratégia de sobrevivência, definimos

$$
\begin{gathered}
\lambda_{i}^{r}(p):=\inf \left\{\lambda: \mathbb{P}\left[M C^{i}(r, \lambda, p) \text { sobrevive }\right]>0\right\}, \quad \text { para } i=1,2, \\
\text { e } \quad \lambda_{3}^{r}(p, m):=\inf \left\{\lambda: \mathbb{P}\left[M C_{m}^{3}(r, \lambda, p) \text { sobrevive }\right]>0\right\} .
\end{gathered}
$$

Observe que para $i=1,2,3$ e qualquer $m$, quando $0<\lambda_{i}^{r}(p)<\infty$ para $0<p<1$, o gráfico da função $\lambda_{i}^{r}(p)$ divide o espaço dos parâmetros $\lambda \times p$ em duas regiões. Para valores de $(\lambda, p)$ acima da curva $\lambda_{i}^{r}(p)$ o modelo $M C^{i}(r, \lambda, p)$ sobrevive com probabilidade positiva, e para valores de $(\lambda, p)$ abaixo da curva $\lambda_{i}^{r}(p)$ o modelo $M C^{i}(r, \lambda, p)$ torna-se extinto com probabilidade 1.

No seguinte resultado estabelecemos algumas propriedades de $\lambda_{2}^{r}(p)$ e $\lambda_{3}^{r}(p, m)$.

Proposição 3.3.1. Seja $0 \leq r \leq 1 e 0<p<1$. Então,

(i) $0<\lambda_{2}^{r}(p)<\lambda_{3}^{r}(p, m+1)<\lambda_{3}^{r}(p, m)<\infty$, para todo $m \geq 2$. Além disso, $\lambda_{3}^{r}(p, 1)=\infty$.

(ii) $\lim _{m \rightarrow \infty} \lambda_{3}^{r}(p, m)=\lambda_{2}^{r}(p)$.

Em diante assumimos $0<p<1$. Do Teorema 3.2.1 segue que se $r<1$ então $\lambda_{1}^{r}(p)=\infty$, e pela Proposição 3.3.1 obtemos que

$$
\lambda_{2}^{r}(p)<\lambda_{3}^{r}(p, m)<\lambda_{1}^{r}(p)
$$

para todo $m \geq 2$. Assim, quando catástrofes binomiais são possíveis $(r<1)$, a dispersão é sempre uma boa estratégia para a sobrevivência da população, com ou sem restrições espaciais. 
Na ausência de catástrofes binomiais $(r=1)$, ou seja, quando os colapsos são só do tipo catástrofes geométricas, é simples calcular $\lambda_{1}^{1}(p), \lambda_{2}^{1}(p)$ e $\lambda_{3}^{1}(p, m)$. Dos Teoremas 3.2.1, 3.2.2 e 3.2 .4 , temos que

$$
\begin{gathered}
\lambda_{1}^{1}(p)=\frac{1-p}{p}, \\
\lambda_{2}^{1}(p)=\sqrt{\frac{1}{4}+\frac{1-p}{p}}-\frac{1}{2}, \\
\lambda_{3}^{1}(p, m)=\frac{1-m p+\sqrt{(1-m p)^{2}+4 m(m-1) p(1-p)}}{2 p(m-1)} .
\end{gathered}
$$

Nesse caso, temos também que $\lambda_{2}^{1}(p)<\lambda_{1}^{1}(p)$. No entanto, podemos ver que a dispersão nem sempre é a melhor estratégia de sobrevivência, veja a Figura 3.1. Observe que

$$
\lambda_{3}^{1}(p, m) \leq \lambda_{1}^{1}(p) \Longleftrightarrow p \leq 1-\frac{1}{m-1}
$$

Portanto, quando os colapsos são sempre do tipo catástrofe geométrica, a existência de vantagem ou não da dispersão como estratégia de sobrevivência depende das restrições espaciais $(m)$ e da probabilidade $(p)$ de um indivíduo exposto a um colapso sobreviver. Ver Figura 3.2.
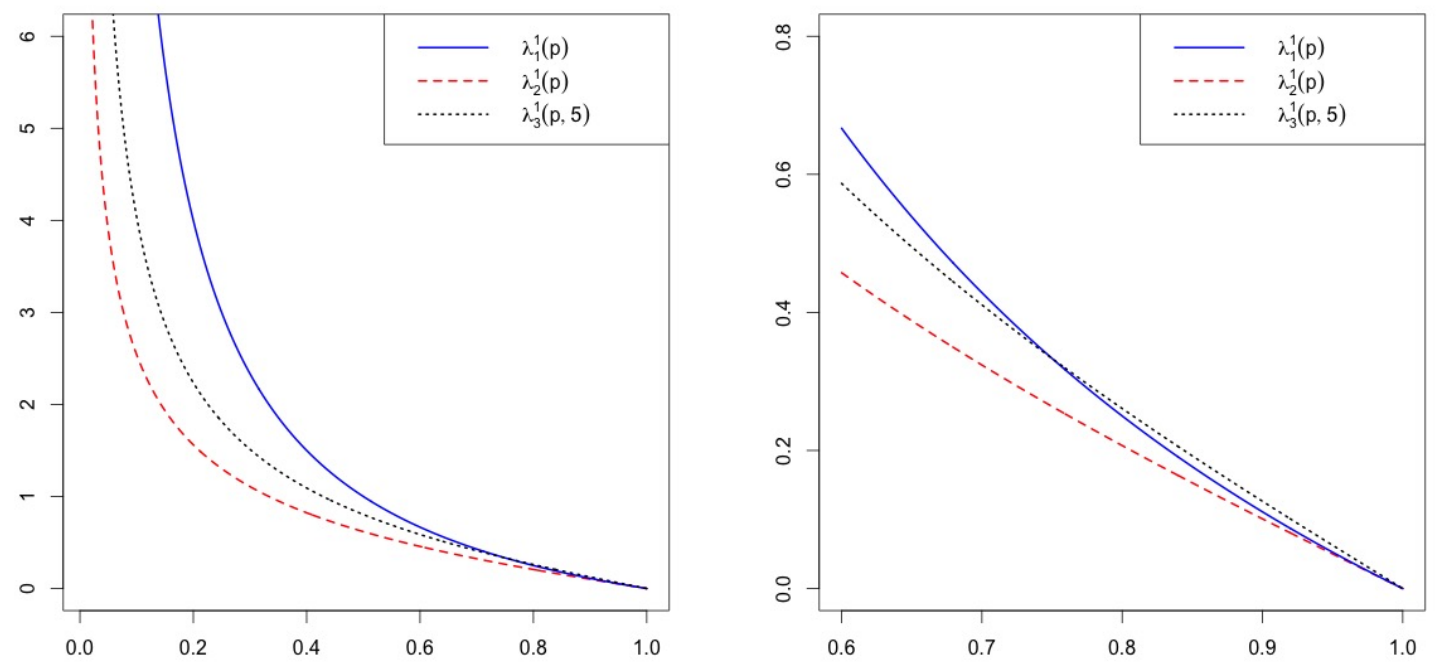

Figura 3.1: Gráficos de $\lambda_{1}^{1}(p), \lambda_{2}^{1}(p), \lambda_{3}^{1}(p, 5)$ 


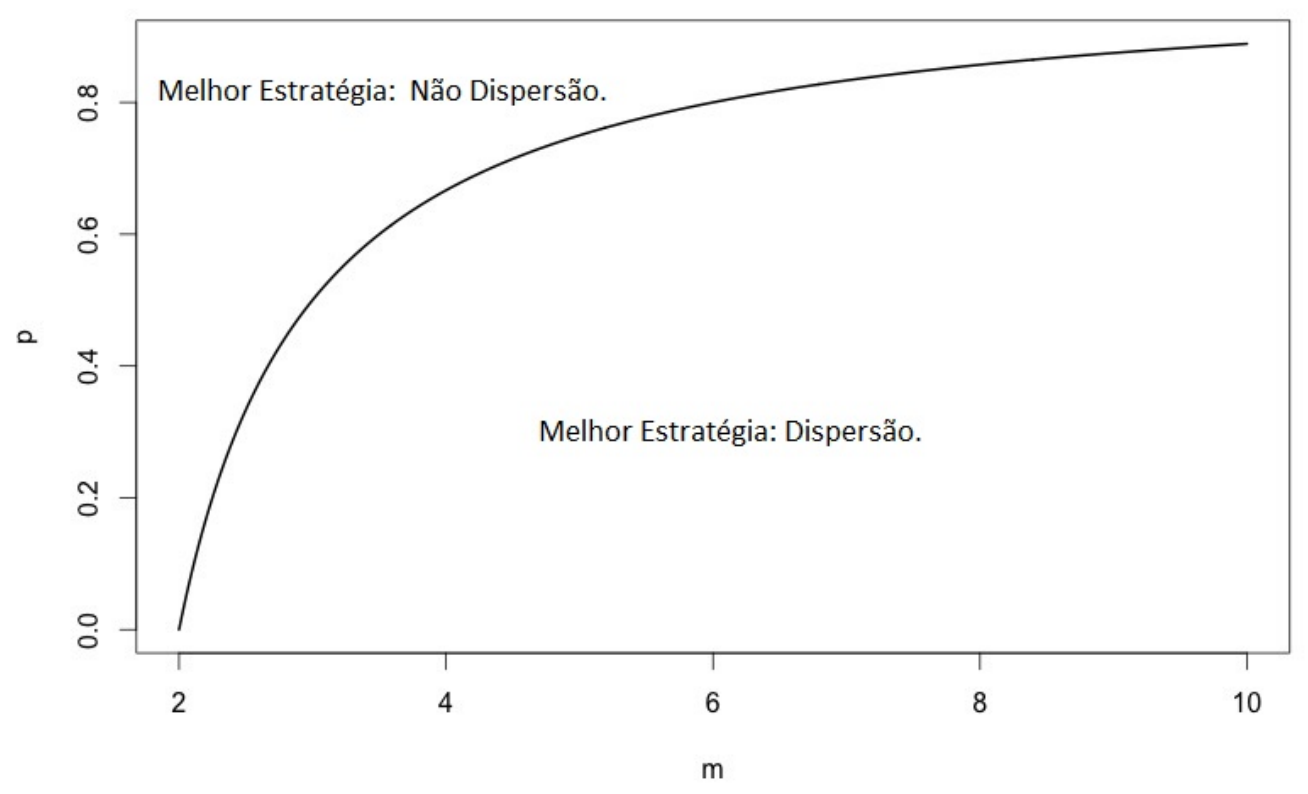

Figura 3.2: Curva $p=1-(m-1)^{-1}$. Melhor estratégia para sobrevivência no caso $r=1$ segundo as restrições espaciais $(m)$ e a probabilidade individual de sobreviver a um colapso $(p)$.

\subsection{Provas}

O Teorema 3.2.1 é consequência dos Teoremas 3.1 e 3.2 em Artalejo et al. [1]. Eles trabalham fortemente com as funções geradoras de momentos da primeira excursão ao 0 (o estado vazio) quando os processos (catástrofes binomiais e geométricas) começam com 1 indivíduo. Aqui nós apresentamos uma prova alternativa para $r<1$, usando o Teorema de Foster, o qual enunciamos a seguir. Para uma prova do Teorema de Foster ver Fayolle et. al. [10, Teorema 2.2.3].

Teorema 3.4.1 (Teorema de Foster). Seja $\left(W_{n}\right)_{n \geq 0}$ uma cadeia de Markov irredutivel e aperiódica com espaço de estados enumerável $\mathcal{A}=\left\{\alpha_{i}, i \geq 0\right\}$. Então, $\left(W_{n}\right)_{n \geq 0}$ é ergódica se e somente se existe uma função positiva $f(\alpha), \alpha \in \mathcal{A}$, um número $\epsilon>0$ e um conjunto finito $A \subset \mathcal{A}$ tal que

$$
\begin{gathered}
\mathbb{E}\left[f\left(W_{n+1}\right)-f\left(W_{n}\right) \mid W_{n}=\alpha_{j}\right] \leq-\epsilon, \quad \alpha_{j} \notin A, \\
\mathbb{E}\left[f\left(W_{n+1}\right) \mid W_{n}=\alpha_{i}\right]<\infty, \quad \alpha_{i} \in A .
\end{gathered}
$$

Apresentamos agora a prova do Teorema 3.2.1.

Prova do Teorema 3.2.1. Seja $\left\{Y_{n}\right\}_{n \geq 0}$ a cadeia de Markov a tempo discreto imersa 
no processo $M C^{1}(r, \lambda, p)$. As probabilidades de transição de $\left\{Y_{n}\right\}_{n \geq 0}$ são dadas por:

$$
\begin{array}{ll}
P_{i, i+1}=\frac{\lambda}{\lambda+1}, & i \geq 0, \\
P_{i, j}=\frac{r \mu_{i j}^{G}+(1-r) \mu_{i j}^{B}}{\lambda+1}, & 0 \leq j \leq i .
\end{array}
$$

Ergocidade de $\left\{Y_{n}\right\}$ implica que o tempo até à extinção de $M C^{1}(r, \lambda, p)$ tem média finita.

Observe que a cadeia $\left\{Y_{n}\right\}$ é irredutível e aperiódica. Usaremos o Teorema de Foster para mostrar que $\left\{Y_{n}\right\}_{n \geq 0}$ é ergódica quando $0 \leq r<1,0<p<1$ e $\lambda>0$. Considere a função $f: \mathbb{N} \rightarrow \mathbb{R}^{+}$definida por $f(i)=i+1, \epsilon>0$ e o conjunto

$$
A:=\left\{i \in \mathbb{N}: \frac{\lambda-i(1-r) q}{1+\lambda}-\frac{r q\left(1-q^{i}\right)}{p(1+\lambda)}>-\epsilon\right\} .
$$

Para $0 \leq r<1,0<p<1$ e $\lambda>0$, o conjunto $A$ é finito. Além disso, temos que

- $\mathbb{E}\left[f\left(Y_{n+1}\right)-f\left(Y_{n}\right) \mid Y_{n}=i\right]=\sum_{j=0}^{i+1}[f(j)-f(i)] P_{i, j}$

$$
\begin{aligned}
& =\frac{\lambda-i(1-r) q}{1+\lambda}-\frac{r q\left(1-q^{i}\right)}{p(1+\lambda)} \\
& \leq-\epsilon \text { para } i \notin A .
\end{aligned}
$$

- $\mathbb{E}\left[f\left(Y_{n+1}\right) \mid Y_{n}=i\right]=\frac{i(\lambda+r q+p)+2 \lambda+r+1}{1+\lambda}-\frac{r\left(1-q^{i+1}\right)}{p(1+\lambda)}$

$$
<\infty \quad \text { para } i \in A \text {. }
$$

Donde, pelo Teorema de Foster segue que $Y_{n}$ é ergódica. Isto conclui a prova.

Visando a prova dos demais resultados definimos o seguinte processo auxiliar.

\section{Processo Auxiliar $\left(Z_{n}^{r, i}\right)_{n \geq 0}$ :}

Considere os modelos $M C^{2}(r, \lambda, p)$ e $M C^{3}(r, \lambda, p)$. Como dito anteriormente, cada um desses modelos inicia com uma única colônia (um indivíduo). Então, definimos $Z_{0}^{r, i}=1$ para $i=2,3$, o número de colônias presentes no tempo 0 em cada modelo. Esta colônia nós chamamos de geração 0 . Após o colapso da colônia, um número aleatório de novas 
colônias são criadas. Denotemos este número aleatório por $Z_{1}^{r, i}$. Esta é a primeira geração de colônias. Todas as colônias da primeira geração dá nascimentos (em tempos aleatórios diferentes) a um número aleatório de novas colônias. Essas novas colônias formam a segunda geração de colônias e sua quantidade é dada por $Z_{2}^{r, i}$. Em geral, para $n \geq 1$, se $Z_{n-1}^{r, i}=0$ então $Z_{n}^{r, i}=0$. Por outro lado, se $Z_{n-1}^{r, i} \geq 1$ então $Z_{n}^{r, i}$ é o número de colônias geradas pelas colônias da geração $n-1$.

Nós afirmamos que $\left\{Z_{n}^{r, i}\right\}_{n \in \mathbb{N}}$ é um processo de ramificação Galton-Watson. Isto é verdade já que o número de descendentes de diferentes colônias do processo $M C^{i}(r, \lambda, p)$ têm a mesma distribuição e são independentes.

Observação 3.4.1. Para $i=2,3$, observe que o processo $M C^{i}(r, \lambda, p)$ morre se e somente se o processo $\left\{Z_{n}^{r, i}\right\}_{n \in \mathbb{N}}$ morre. Além disso, como $\left\{Z_{n}^{r, i}\right\}_{n \in \mathbb{N}}$ é um processo de ramificação Galton-Watson, este sobrevive com probabilidade positiva se e somente se $\mathbb{E}\left[Z_{1}^{r, i}\right]>1$. Por fim, a probabilidade de extinção de $\left\{Z_{n}^{r, i}\right\}_{n \in \mathbb{N}}$ é a menor solução não negativa de $\phi_{r, i}(s)=s$, onde $\phi_{r, i}(s)$ é a função geradora de probabilidade de $Z_{1}^{r, i}$.

Lema 3.4.1. A função geradora de probabilidade de $Z_{1}^{r, 2}$ é dada por:

$$
\phi_{r, 2}(s)=\frac{1}{1+\lambda p}\left[q+\frac{r(\lambda+1) p s}{1+\lambda-\lambda s}+\frac{(1-r)(\lambda+1) p s}{1+\lambda p-\lambda p s}\right]
$$

$e$

$$
\mathbb{E}\left[Z_{1}^{r, 2}\right]=\frac{p(\lambda+1)^{2} r}{\lambda p+1}+p(\lambda+1)(1-r)
$$

Demonstração. Lembremos que $Z_{1}^{r, 2}$ é o número de sobreviventes ao colapso de uma colônia no modelo $M C^{2}(r ; \lambda ; p)$. Denotemos $Z_{B}:=Z_{1}^{0,2}$ e $Z_{G}:=Z_{1}^{1,2}$. Iniciamos mostrando que

$$
\begin{aligned}
& \mathbb{P}\left[Z_{B}=k\right]= \begin{cases}\frac{1+\lambda}{\lambda(1+\lambda p)}\left(\frac{\lambda p}{1+\lambda p}\right)^{k}, & k \geq 1 \\
\frac{q}{1+\lambda p}, & k=0 .\end{cases} \\
& \mathbb{P}\left[Z_{G}=k\right]= \begin{cases}\frac{p}{1+\lambda p}\left(\frac{\lambda}{1+\lambda}\right)^{k-1}, & k \geq 1 \\
\frac{q}{1+\lambda p}, & k=0 .\end{cases}
\end{aligned}
$$


Para $k=0$, note que $\mathbb{P}\left[Z_{B}=0\right]=\mathbb{P}\left[Z_{G}=0\right]$ com

$$
\mathbb{P}\left[Z_{B}=0\right]=\int_{0}^{\infty} e^{-t} \sum_{n=0}^{\infty} \frac{e^{-\lambda t}(\lambda t)^{n}}{n !} q^{n+1} d t=q \int_{0}^{\infty} e^{-(\lambda p+1) t} d t=\frac{q}{1+\lambda p}
$$

Para $k \geq 1$,

$$
\begin{aligned}
\mathbb{P}\left[Z_{B}=k\right] & =\int_{0}^{\infty} e^{-t} \sum_{n=k-1}^{\infty} \frac{e^{-\lambda t}(\lambda t)^{n}}{n !}\left(\begin{array}{c}
n+1 \\
k
\end{array}\right) p^{k} q^{n+1-k} d t \\
& =q\left(\frac{p}{q}\right)^{k} \sum_{n=k-1}^{\infty}\left(\begin{array}{c}
n+1 \\
k
\end{array}\right) \frac{(\lambda q)^{n}}{n !} \int_{0}^{\infty} e^{-(\lambda+1) t} t^{n} d t \\
& =q\left(\frac{p}{q}\right)^{k} \sum_{n=k-1}^{\infty}\left(\begin{array}{c}
n+1 \\
k
\end{array}\right) \frac{(\lambda q)^{n}}{n !} \frac{\Gamma(n+1)}{(\lambda+1)^{n+1}} \\
& =\frac{q}{\lambda+1}\left(\frac{p}{q}\right)^{k} \sum_{n=k-1}^{\infty}\left(\begin{array}{c}
n+1 \\
k
\end{array}\right)\left(\frac{\lambda q}{\lambda+1}\right)^{n} \\
& =\frac{q}{\lambda+1}\left(\frac{p}{q}\right)^{k}\left(\frac{\lambda q}{\lambda+1}\right)^{k-1} \sum_{j=0}^{\infty}\left(\begin{array}{c}
j+k \\
k
\end{array}\right)\left(\frac{\lambda q}{\lambda+1}\right)^{j} \\
& =\frac{q}{\lambda+1}\left(\frac{p}{q}\right)^{k}\left(\frac{\lambda q}{\lambda+1}\right)^{k-1}\left(1-\frac{\lambda q}{\lambda+1}\right)^{-(k+1)} \\
& =\frac{1+\lambda}{\lambda(1+\lambda p)}\left(\frac{\lambda p}{1+\lambda p}\right)^{k} .
\end{aligned}
$$

Similarmente,

$$
\begin{aligned}
\mathbb{P}\left[Z_{G}=k\right] & =\int_{0}^{\infty} e^{-t} \sum_{n=k-1}^{\infty} \frac{e^{-\lambda t}(\lambda t)^{n}}{n !} p q^{n+1-k} d t \\
& =p q^{1-k} \sum_{n=k-1}^{\infty} \frac{(q \lambda)^{n}}{n !} \int_{0}^{\infty} e^{-(\lambda+1) t} t^{n} d t \\
& =p q^{1-k} \sum_{n=k-1}^{\infty} \frac{(q \lambda)^{n}}{n !} \frac{\Gamma(n+1)}{(\lambda+1)^{n+1}} \\
& =\frac{p q^{1-k}}{\lambda+1} \sum_{n=k-1}^{\infty}\left(\frac{q \lambda}{\lambda+1}\right)^{n} \\
& =\frac{p q^{1-k}}{\lambda+1}\left(\frac{q \lambda}{\lambda+1}\right)^{k-1} \sum_{j=0}^{\infty}\left(\frac{q \lambda}{\lambda+1}\right)^{j} \\
& =\frac{p}{1+\lambda p}\left(\frac{\lambda}{\lambda+1}\right)^{k-1} .
\end{aligned}
$$


Usando (3.4.1) obtemos que

$$
\begin{aligned}
\phi_{B}(s) & =\sum_{k \geq 0} \mathbb{P}\left[Z_{B}=k\right] s^{k} \\
& =\frac{q}{1+\lambda p}+\frac{1+\lambda}{\lambda(1+\lambda p)} \sum_{k \geq 1}\left(\frac{\lambda p s}{1+\lambda p}\right)^{k} \\
& =\frac{1}{1+\lambda p}\left[q+\frac{(\lambda+1) p s}{1+\lambda p-\lambda p s}\right] .
\end{aligned}
$$

Da mesma forma, usando (3.4.2) obtemos que

$$
\begin{aligned}
\phi_{G}(s) & =\sum_{k \geq 0} \mathbb{P}\left[Z_{G}=k\right] s^{k} \\
& =\frac{q}{1+\lambda p}+\frac{s p}{1+\lambda p} \sum_{k \geq 1}\left(\frac{\lambda s}{1+\lambda}\right)^{k-1} \\
& =\frac{1}{1+\lambda p}\left[q+\frac{(\lambda+1) p s}{1+\lambda-\lambda s}\right] .
\end{aligned}
$$

Finalmente, o resultado desejado segue observando que

$$
\phi_{r, 2}(s)=r \phi_{G}(s)+(1-r) \phi_{B}(s),
$$

e calculando $\mathbb{E}\left[Z_{1}^{r, 2}\right]=\phi_{r, 2}^{\prime}(1)$.

Lema 3.4.2. A função geradora de probabilidade de $Z_{1}^{r, 3}$ é dada por:

$$
\psi_{r, 3}(s)=r \psi_{G}(s)+(1-r) \psi_{B}(s)
$$

onde

$$
\begin{aligned}
& \psi_{B}(s):=\frac{q}{1+\lambda p}+\frac{m(1+\lambda)}{\lambda} \sum_{k=1}^{m}\left(\begin{array}{c}
m \\
k
\end{array}\right)\left[\frac{-\lambda p s}{m(1+\lambda p)}\right]^{k} \sum_{j=0}^{k}\left(\begin{array}{c}
k \\
j
\end{array}\right) \frac{(-1)^{j} j^{k}}{m(1+\lambda p)-\lambda p j}, \\
& \psi_{G}(s):=\frac{q}{1+\lambda p}+\frac{(1+\lambda) p s}{\lambda p+1} \sum_{k=1}^{m}\left(\begin{array}{c}
m \\
k
\end{array}\right)\left[\frac{-\lambda s}{m(1+\lambda)}\right]^{k-1} \sum_{j=0}^{k}\left(\begin{array}{c}
k \\
j
\end{array}\right) \frac{(-1)^{j-1} j^{k}}{m(1+\lambda)-\lambda j} .
\end{aligned}
$$

Além disso,

$$
\mathbb{E}\left[Z_{1}^{r, 3}\right]=\frac{m p(\lambda+1)^{2} r}{(m+\lambda)(\lambda p+1)}+\frac{m p(\lambda+1)(1-r)}{m+\lambda p} .
$$

Demonstração. Considere o modelo $M C_{m}^{3}(r, \lambda, p)$ iniciando com uma única colônia em algum vértice $x$ de $\mathcal{G}$. Seja $Z$ o número de indivíduos que sobrevivem nesta colônia 
quando ela colapsa e $W:=Z_{1}^{r, 3}$ o número de novas colônias criadas pelos sobreviventes após o colapso.

Da definição de $M C_{m}^{3}(r, \lambda, p)$ segue que

$$
\mathbb{P}[Z=j]=r \mathbb{P}\left[Z_{G}=j\right]+(1-r) \mathbb{P}\left[Z_{B}=j\right],
$$

onde $Z_{B}$ e $Z_{G}$ são as variáveis definidas em (3.4.1) e (3.4.2), respectivamente. Por outro lado, para $k \in\{1, \ldots, m\}$ e $j \geq k$, observe que

$$
\mathbb{P}[W=k \mid Z=j]=\left(\begin{array}{c}
m \\
k
\end{array}\right) \frac{T(j, k)}{m^{j}},
$$

onde $T(j, k)=\sum_{i=0}^{k}\left(\begin{array}{c}k \\ i\end{array}\right)(-1)^{i}(k-i)^{j}$ é o número de funções sobrejetoras com domínio em um conjunto de $j$ elementos e contradomínio em um conjunto de $k$ elementos. Assim, para $k \in\{1, \ldots, m\}$,

$$
\begin{aligned}
\mathbb{P}[W=k]= & r \sum_{j=k}^{\infty}\left(\begin{array}{c}
m \\
k
\end{array}\right) \frac{T(j, k)}{m^{j}} \mathbb{P}\left[Z_{G}=j\right] \\
& +(1-r) \sum_{j=k}^{\infty}\left(\begin{array}{c}
m \\
k
\end{array}\right) \frac{T(j, k)}{m^{j}} \mathbb{P}\left[Z_{B}=j\right] .
\end{aligned}
$$

- Usando (3.4.1), temos que

$$
\begin{aligned}
\sum_{j=k}^{\infty}\left(\begin{array}{c}
m \\
k
\end{array}\right) \frac{T(j, k)}{m^{j}} \mathbb{P}\left[Z_{B}=j\right] \\
=\left(\begin{array}{c}
m \\
k
\end{array}\right) \frac{1+\lambda}{\lambda(\lambda p+1)} \sum_{j=k}^{\infty}\left[\frac{\lambda p}{m(\lambda p+1)}\right]^{j} T(j, k) \\
=\left(\begin{array}{c}
m \\
k
\end{array}\right) \frac{1+\lambda}{\lambda(\lambda p+1)}\left[\frac{\lambda p}{m(\lambda p+1)}\right]^{k} \sum_{j=0}^{\infty}\left[\frac{\lambda p}{m(\lambda p+1)}\right]^{j} T(j+k, k) \\
=\left(\begin{array}{c}
m \\
k
\end{array}\right) \frac{1+\lambda}{\lambda(\lambda p+1)}\left[\frac{\lambda p}{m(\lambda p+1)}\right]^{k} \sum_{j=0}^{\infty}\left[\frac{\lambda p}{m(\lambda p+1)}\right]^{j} \sum_{i=0}^{k}\left(\begin{array}{c}
k \\
i
\end{array}\right)(-1)^{i}(k-i)^{j+k} \\
=\left(\begin{array}{c}
m \\
k
\end{array}\right) \frac{1+\lambda}{\lambda(\lambda p+1)}\left[\frac{\lambda p}{m(\lambda p+1)}\right]^{k} \sum_{i=0}^{k}\left(\begin{array}{c}
k \\
i
\end{array}\right)(-1)^{i}(k-i)^{k} \sum_{j=0}^{\infty}\left[\frac{\lambda p(k-i)}{m(\lambda p+1)}\right]^{j} \\
=\left(\begin{array}{c}
m \\
k
\end{array}\right) \frac{m(1+\lambda)}{\lambda}\left[\frac{\lambda p}{m(\lambda p+1)}\right]^{k} \sum_{i=0}^{k}\left(\begin{array}{c}
k \\
i
\end{array}\right) \frac{(-1)^{i}(k-i)^{k}}{m(\lambda p+1)-\lambda p(k-i)} .
\end{aligned}
$$

- Similarmente, usando (3.4.2), temos que 


$$
\begin{aligned}
\sum_{j=k}^{\infty}\left(\begin{array}{c}
m \\
k
\end{array}\right) \frac{T(j, k)}{m^{j}} \mathbb{P}\left[Z_{G}=j\right] \\
=\left(\begin{array}{c}
m \\
k
\end{array}\right) \frac{p}{m(\lambda p+1)} \sum_{j=k}^{\infty}\left[\frac{\lambda}{m(\lambda+1)}\right]^{j-1} T(j, k) \\
=\left(\begin{array}{c}
m \\
k
\end{array}\right) \frac{p}{m(\lambda p+1)}\left[\frac{\lambda}{m(\lambda+1)}\right]^{k-1} \sum_{j=0}^{\infty}\left[\frac{\lambda}{m(\lambda+1)}\right]^{j} T(j+k, k) \\
=\left(\begin{array}{c}
m \\
k
\end{array}\right) \frac{p}{m(\lambda p+1)}\left[\frac{\lambda}{m(\lambda+1)}\right]^{k-1} \sum_{j=0}^{\infty}\left[\frac{\lambda}{m(\lambda+1)}\right]^{j} \sum_{i=0}^{k}\left(\begin{array}{c}
k \\
i
\end{array}\right)(-1)^{i}(k-i)^{j+k} \\
=\left(\begin{array}{c}
m \\
k
\end{array}\right) \frac{p}{m(\lambda p+1)}\left[\frac{\lambda}{m(\lambda+1)}\right]^{k-1} \sum_{i=0}^{k}\left(\begin{array}{c}
k \\
i
\end{array}\right)(-1)^{i}(k-i)^{k} \sum_{j=0}^{\infty}\left[\frac{\lambda(k-i)}{m(\lambda+1)}\right]^{j} \\
=\left(\begin{array}{c}
m \\
k
\end{array}\right) \frac{(1+\lambda) p}{\lambda p+1}\left[\frac{\lambda}{m(1+\lambda)}\right]^{k-1} \sum_{i=0}^{k}\left(\begin{array}{c}
k \\
i
\end{array}\right) \frac{(-1)^{i}(k-i)^{k}}{m(1+\lambda)-\lambda(k-i)} .
\end{aligned}
$$

Finalmente, observe que $\mathbb{P}[W=0]=\mathbb{P}[Z=0]=q /(1+\lambda p)$. Junto com (3.4.4),(3.4.5) e (3.4.6) obtemos a expressão desejada da função geradora de probabilidade de $W$.

Para calcular $\mathbb{E}[W]$ enumeramos cada vizinho do vértice $x$ de 1 até $m$. Daí escrevemos $W=\sum_{i=1}^{m} I_{i}$, onde $I_{i}$ é a função indicadora do evento \{Uma nova colônia é criada no $i$-ésimo vizinho de $x\}$. Portanto,

$$
\mathbb{E}[W]=\sum_{i=1}^{m} \mathbb{P}\left[I_{i}=1\right]=m \mathbb{P}\left[I_{1}=1\right]
$$

Observe que

$$
\mathbb{P}\left[I_{1}=1 \mid Z=k\right]=1-\left(\frac{m-1}{m}\right)^{k}
$$

logo, usando (3.4.3) nós temos

$$
\begin{aligned}
\mathbb{P}\left[I_{1}=1\right]= & r \sum_{k=1}^{\infty}\left[1-\left(\frac{m-1}{m}\right)^{k}\right] \mathbb{P}\left[Z_{G}=k\right] \\
& +(1-r) \sum_{k=1}^{\infty}\left[1-\left(\frac{m-1}{m}\right)^{k}\right] \mathbb{P}\left[Z_{B}=k\right] .
\end{aligned}
$$


Substituindo (3.4.1) e (3.4.2) em (3.4.8) após alguns cálculos obtemos

$$
\mathbb{P}\left[I_{1}=1\right]=\frac{p(\lambda+1)^{2} r}{(m+\lambda)(\lambda p+1)}+\frac{p(\lambda+1)(1-r)}{m+\lambda p} .
$$

Finalmente, substituindo (3.4.9) em (3.4.7) nós obtemos o resultado desejado.

Provas dos Teoremas 3.2.2 e 3.2.4. Da Observação 3.4 .1 temos que $M C^{i}(r, \lambda, p)$ sobrevive se e somente se $\mathbb{E}\left[Z_{n}^{r, i}\right]>1$. Dos Lemas 3.4.1 e 3.4.2 seguem os resultados.

Provas dos Teoremas 3.2.3 e 3.2.5. Da Observação 3.4.1 temos que as probabilidades de extinção, $\rho_{2}(r)$ e $\rho_{3}(r)$, dos processos $M C^{2}(r, \lambda, p)$ e $M C_{m}^{3}(r, \lambda, p)$, são as menores soluções em $[0,1]$ de $\phi_{r, i}(s)=s$ para $i=2$ e 3, respectivamente. Dos Lemas 3.4.1 e 3.4.2 seguem os resultados desejados.

Prova da Proposição 3.3.1 (i). Primeiro definimos as seguintes funções

$$
\begin{aligned}
f_{m}(\lambda) & :=\frac{m p(1+\lambda)^{2} r}{(m+\lambda)(\lambda p+1)}+\frac{m p(1+\lambda)(1-r)}{m+\lambda p} \\
f(\lambda) & :=\frac{p(\lambda+1)^{2} r}{\lambda p+1}+p(\lambda+1)(1-r)
\end{aligned}
$$

Dos Teoremas 3.2.2 e 3.2.4 segue que

$$
\begin{gathered}
\lambda_{2}^{r}(p)=\inf \{\lambda: f(\lambda)>1\}, \\
\lambda_{3}^{r}(p, m)=\inf \left\{\lambda: f_{m}(\lambda)>1\right\} .
\end{gathered}
$$

Observe que as $f_{m}$ e $f$ são funções continuas em $[0, \infty)$, com $f_{m}(0)=f(0)=p<1$, $\lim _{\lambda \rightarrow \infty} f(\lambda)=\infty$ e $\lim _{\lambda \rightarrow \infty} f_{m}(\lambda)=m$.

Além disso, $\left\{f_{m}\right\}_{m \geq 1}$ é uma sequência estritamente crescente de funções estritamente crescentes em $(0, \infty)$ tais que $\lim _{m \rightarrow \infty} f_{m}(\lambda)=f(\lambda)$. Também, $f$ é estritamente crescente.

Logo, pelo Teorema do Valor Intermediário e a definição de $\lambda_{2}^{r}(p)$ e $\lambda_{3}^{r}(p, m)$ temos que $0<\lambda_{2}^{r}(p)<\infty, 0<\lambda_{3}^{r}(p, m)<\infty$ para $m \geq 2$, e $\lambda_{3}^{r}(p, 1)=\infty$. Além disso,

$$
\begin{aligned}
& f(\lambda)=1 \Longleftrightarrow \lambda=\lambda_{2}^{r}(p), \\
& f_{m}(\lambda)=1 \quad \Longleftrightarrow \lambda=\lambda_{3}^{r}(p, m) .
\end{aligned}
$$


Suponha, por contradição, que $\lambda_{3}^{r}(p, m) \leq \lambda_{3}^{r}(p, m+1)$ para algum $m \geq 2$. Do anterior temos que

$$
1=f_{m}\left(\lambda_{3}^{r}(p, m)\right) \leq f_{m}\left(\lambda_{3}^{r}(p, m+1)\right)<f_{m+1}\left(\lambda_{3}^{r}(p, m+1)\right)=1, \quad \text { Absurdo! }
$$

Portanto, $\lambda_{3}^{r}(p, m+1)<\lambda_{3}^{r}(p, m)$, para todo $m \geq 1$. Similarmente mostra-se que $\lambda_{2}^{r}(p)<\lambda_{3}^{r}(p, m)$ para todo $m \geq 1$.

Prova da Proposição 3.3.1 (ii). Nós restringimos o domínio das funções $f_{m}$ e $f$ a $\left[0, \lambda_{3}^{r}(p, 2)\right]$. Como as $f_{m}$ e $f$ são funções continuas, $\lim _{m \rightarrow \infty} f_{m}=f$ e $f_{m}(\lambda)<f_{m+1}(\lambda)$ para todo $\lambda \in\left[0, \lambda_{3}^{r}(p, 2)\right]$, então, $f_{m}$ converge uniformemente a $f$ em $\left[0, \lambda_{3}^{r}(p, 2)\right]$, ver Rudin [19, Teorema 7.13].

Do item $(i)$ segue que $\lambda_{3}^{r}(p, m) \in\left[0, \lambda_{3}^{r}(p, 2)\right]$ para todo $m \geq 2$, e que existe o limite $\theta:=\lim _{m \rightarrow \infty} \lambda_{3}^{r}(p, m)$. Assim, pela convergência uniforme das $f_{m}$ a $f$, segue que $f(\theta)=\lim _{m \rightarrow \infty} f_{m}\left(\lambda_{3}^{r}(p, m)\right)=1$, ver Rudin [19, Exercício 9 do Capítulo 7]. Finalmente, de (3.4.10) segue o resultado. 


\section{Referências Bibliográficas}

[1] J.R. Artalejo, A. Economou, M.J. Lopez-Herrero. Evaluating growth measures in an immigration process subject to binomial and geometric catastrophes. Mathematical Biosciences and Engineering 4, (4), 573-594 (2007).

[2] L.J. Allen. An Introduction to Stochastic Processes with Applications to Biology, Second Edition. Taylor \& Francis Group. (2010).

[3] L. Belhadji, N. Lanchier. Individual versus cluster recoveries within a spatially structured population. Ann. Appl. Probab. 16, 403-422. (2006).

[4] R. N. Bhattacharya, E. C. Waymire. Stochastic Processes With Applications. Society for Industrial \&3 Applied. (2009).

[5] D. Borrello. On the role of Allee effect and mass migration in survival and extinction of a species. Ann. Appl. Probab. 22, (2), 670-701. (2012).

[6] P.J. Brockwell, J. Gani, S.I. Resnick. Birth, Immigration and Catastrophe Processes. Advances in Applied Probability 14, (4), 709-731. (1982).

[7] P.J. Brockwell. The extinction time for a general birth and death process with catastrophes. Journal of Applied Probability 23, 851-858. (1986).

[8] M. Bramson, R. Durrett. Simple proof of the stability criterion of Gray and Griffeath. Probability Theory and Related Fields 80, 293-298. (1988).

[9] R. Durrett. Oriented Percolation in two dimensions. The Annals of Probability 12 (4), 999-1040. (1984).

[10] G. Fayolle, V. A. Malyshev, M. V. Menshikov. Topics in the Constructive Theory of Countable Markov Chains. Cambridge University Press (1995).

[11] I. Hanski. Metapopulation Ecology. Oxford Univ. Press, Oxford. (1999).

[12] V.V. Junior, F.P. Machado, A. Roldán-Correa. Dispersion as a Survival Strategy. arXiv:1603.00907 (2016).

[13] H.K. Kang, S.M. Krone, C. Neuhauser. Stepping-stone models with extinction and recolonization. Ann. Appl. Probab. 5, 1025-1060. (1995). 
[14] S. Kapodistria, T. Phung-Duc, J. Resing. Linear birth/immigration-death process with binomial catastrophes. Probability in the Engineering and Informational Sciences 30, (1), 79-111. (2016).

[15] S.M. Krone. The two-stage contact process. Ann. Appl. Probab. 9, 331-351. (1999).

[16] T. Liggett. Interacting particle systems. Springer-Verlag. (1985).

[17] Y.L. Luke. The Special Functions and Their Approximations. Academic Press, New York vol. 1. (1969).

[18] F.P. Machado, A. Roldán-Correa, R. Schinazi. Colonization and Collapse. arXiv:1510.02704 (2015).

[19] W. Rudin. Principles of Mathematical Analysis, Third Edition. McGraw-Hill,Inc. (1976).

[20] R. Schinazi. Does random dispersion help survival? Journal of Statistical Physics, 159, (1), 101-107 (2015).

[21] R. Schinazi. Mass extinctions: an alternative to the Allee effect. Ann. Appl. Probab., 15, (1B), 984-991 (2005).

[22] R. Schinazi. On the role of social clusters in the transmission of infectious diseases. Theoretical Population Biology, 61, 163-169 (2002). 